6-1988

\title{
Atomic Electrons in Strong Magnetic Fields: Transition from Elliptical to Helical Behavior.
}

John B. Delos

William \& Mary, jbdelos@wm.edu

Stephen Knudson

William \& Mary

Shena Sikora

William \& Mary

Robert Leonard Waterland

William \& Mary

S. Whitworth

William \& Mary

Follow this and additional works at: https://scholarworks.wm.edu/aspubs

Part of the Physics Commons

\section{Recommended Citation}

Delos, John B.; Knudson, Stephen; Sikora, Shena; Waterland, Robert Leonard; and Whitworth, S., Atomic Electrons in Strong Magnetic Fields: Transition from Elliptical to Helical Behavior. (1988). Physical Review A, 37(12), 4582-4598.

https://doi.org/10.1103/PhysRevA.37.4582

This Article is brought to you for free and open access by the Arts and Sciences at W\&M ScholarWorks. It has been accepted for inclusion in Arts \& Sciences Articles by an authorized administrator of W\&M ScholarWorks. For more information, please contact scholarworks@wm.edu. 


\title{
Atomic electrons in strong magnetic fields: Transition from elliptical to helical behavior
}

\author{
J. B. Delos, S. K. Knudson, S. D. Sikora, R. L. Waterland, and S. Whitworth \\ Department of Physics, College of William and Mary, Williamsburg, Virginia 23185 \\ and Department of Chemistry, College of William and Mary, Williamsburg, Virginia 23185
}

(Received 30 November 1987)

\begin{abstract}
The behavior of an atomic electron in a static magnetic field strong enough to correspond to the transition regime is examined. The field strength is characterized by the parameter $\hat{L}$, the effective component of angular momentum. A Floquet-Mathieu analysis shows that the bifurcation of classical trajectories into elliptical and helical families is related to the 2:1 resonance which occurs at $\hat{L}=\hat{L}_{T}$. Quantum mechanics gives an avoided crossing at $\hat{L}_{T}$; we examine the nature of the wave functions as $\hat{L}$ passes through the resonance. Semiclassical calculations accurately reproduce the quantum eigenvalues and produce trajectories which underlie the quantum wave functions. The avoided crossing is expressed in semiclassical terms as a switch between elliptical and helical families. The bifurcation of the classical motion means that, at the primitive semiclassical level, some states may be missed and others may be generated in both elliptical and helical representations.
\end{abstract}

\section{INTRODUCTION}

This is one of a series of papers dealing with classical and semiclassical descriptions of atoms in strong magnetic fields. ${ }^{1}$ In Ref. 1 (c), we presented an overview of the types of behavior that occur at various energies and field strengths. It was shown that for weak fields the trajectories are Kepler ellipses with slowly varying orbital parameters, and that for strong fields the trajectories have a helical structure. For intermediate field strengths, at sufficiently high energies the trajectories are irregular or chaotic, and apparently fill the energy shell. Finally, at these intermediate field strengths but low energies, there is a "transition regime" in which the trajectories are regular and have a character that is somehow intermediate between elliptical and helical. A preliminary analysis of the trajectories and quantum states in this regime was given in that paper. In the present paper we analyze this regime in much more detail. We show that the transition regime is associated with a 2:1 frequency resonance, and we display the effects of this resonance on the trajectories and on the energy spectrum.

Similar resonances are sometimes found in vibrational spectra of polyatomic molecules. Consider, for example, the stretching motions of a triatomic molecule. There are two degrees of freedom, and if we carry out the usual normal-mode analysis, expanding the potential-energy and kinetic-energy functions about the equilibrium point, we obtain in lowest order the harmonic-oscillator approximation to the energy spectrum

$$
E_{n_{1} n_{2}}=\left(n_{1}+1 / 2\right) h v_{1}+\left(n_{2}+1 / 2\right) h v_{2} \text {. }
$$

If it should happen that the masses and force constants combine in such a way that $v_{2}=2 v_{1}$, then this formula predicts a series of degeneracies. The lowest two energy levels are nondegenerate, the next two are doubly degenerate, the next two triply degenerate, then two quadruply degenerate, and so on. Higher-order terms in the Taylor expansion of the potential energy strongly couple the degenerate states, and the degeneracies are split so that the resulting sequence of eigenvalues is very complicated. Such a situation is known to molecular spectroscopists as "Fermi resonance."

It is interesting to read Herzberg's discussion of Fermi resonance, ${ }^{2}$ to see what was known about the subject in 1945. He mentions Fermi resonances repeatedly, but mainly to make the point that simple formulas such as (1.1) break down in such cases. He does not give any adequate description of the spectrum when such resonances are present. He also mentions that "a resonance phenomenon occurs also in a classical treatment of the vibrations. . . [I] $f$ at first only $v_{2}$ is excited, after a while only $v_{1}$ will be excited; and after a further interval only $v_{2}$ will be excited, and so on. The situation is much the same as for two coupled pendulums. The motion may be considered as the superposition of two stationary vibrations of somewhat different frequencies." This is partly true, but without computer-generated trajectories, there was no way to get a complete picture of the subject.

The recent resurgence of interest in classical and semiclassical mechanics has led to a burst of activity on the theory of such resonances. ${ }^{3,4}$ However, the question that we consider most fundamental has not yet been answered in a satisfactory way: What, precisely, are the trajectory structures that arise, and what is the relationship between the trajectories and the quantum states?

Noid and his collaborators ${ }^{5}$ have developed simple and powerful techniques for analyzing phase-space structures, and for numerical computation of action variables and semiclassical eigenvalue spectra. Thiele and his coworkers ${ }^{6}$ over a period of years have shown that some properties of trajectories can be understood using Floquet analysis and study of the eigenvalues of Mathieu's equation.

We shall combine these two different sets of ideas to analyze the trajectories and the semiclassical eigenvalue spectra associated with the transition regime. We exam- 
ine exact trajectories and semiclassical eigenstates using the methods of Noid, Koszykowski, and Marcus. ${ }^{3(a)}$ Surprisingly, however, on one central point, we arrive at conclusions that are the opposite of theirs: Noid and coworkers have concluded that primitive semiclassical eigenvalues cross, whereas in our case they avoid crossing. In a later section we adapt and extend the methods of Thiele and co-workers to obtain additional insight into the nature of the bifurcation of trajectories. In this way, we shall not only obtain detailed information about an atomic electron in a strong magnetic field, we shall also obtain a much more complete understanding of the consequences of 2:1 resonances in vibrating systems.

Atomic hydrogen in a magnetic field constitutes a model system which can be studied experimentally. As will be seen, once the atoms have been generated (in a particular $L_{z}$ state), the magnetic field may be used to tune through the resonance region.

\section{COORDINATES, SCALING, HAMILTONIAN}

The details of the construction of the Hamiltonian have been discussed previously; ${ }^{1}$ we include here only enough to make this paper reasonably self-contained. We employ cylindrical coordinates $\rho$ and $z$ in a reference frame rotating at the Larmor frequency about the direction of the magnetic field, which is taken to be the $z$ axis. Let $Z$ be the number of positive charges on the nucleus, $L_{z}$ the $z$ component of the angular momentum, $m$ the mass of the electron, and $B$ the magnitude of the field. Then the parameters $k$ and $\lambda$ may be defined as $k=Z e^{2}$, $\lambda=e^{2} B^{2} / 8 m c^{2}$, and a transformation to scaled variables according to

$$
\begin{aligned}
& \hat{z}=z / \alpha, \quad \hat{\rho}=\rho / \alpha, \\
& \hat{P}_{z}=P_{z} / \beta, \quad \hat{P}_{\rho}=P_{\rho} / \beta, \\
& \hat{t}=t / \gamma,
\end{aligned}
$$

with

$$
\begin{aligned}
& \alpha=(k / 8 \lambda)^{1 / 3}, \\
& \beta=m^{1 / 2} k^{1 / 3}(8 \lambda)^{1 / 6}, \\
& \gamma=(m / 8 \lambda)^{1 / 2}
\end{aligned}
$$

converts the Hamiltonian to the form

$$
\begin{aligned}
\hat{H}=\frac{m H}{\beta^{2}}= & \frac{1}{2}\left(\hat{P}_{\rho}^{2}+\hat{P}_{z}^{2}\right)-\left(\hat{\rho}^{2}+\hat{z}^{2}\right)^{-1 / 2} \\
& +\hat{L}^{2} / 2 \hat{\rho}^{2}+\hat{\rho}^{2} / 8
\end{aligned}
$$

where the only parameter is $\hat{L}$, the effective component of angular momentum,

$$
\hat{L}=L_{z} / \alpha \beta \text {. }
$$

A contour plot of the potential energy for $\hat{L}$ near 1.60 is given in Fig. 1. Unless otherwise noted, for the remainder of this paper the carets will be omitted from the scaled variables, and only scaled variables will be used in equations. The equations of motion take the form

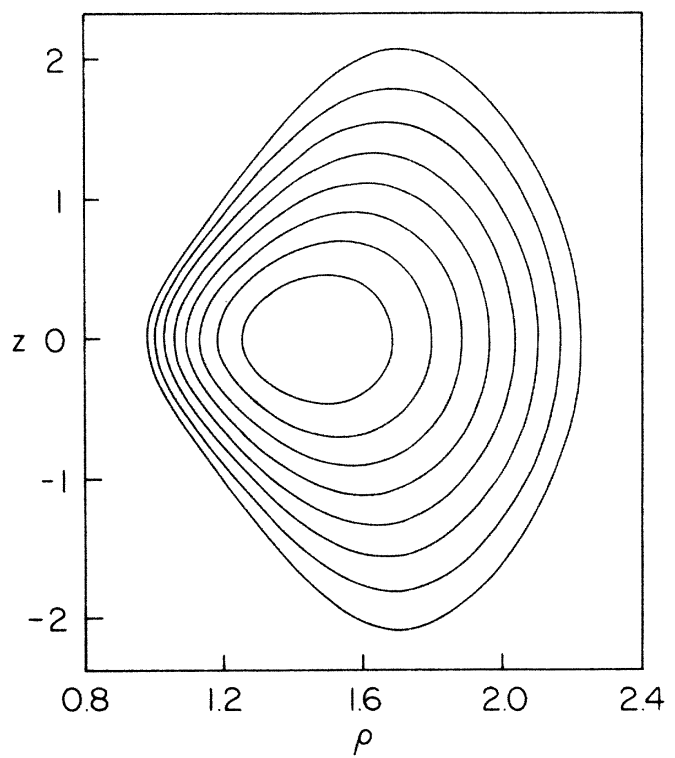

FIG. 1. A contour plot of the effective potential $V=\hat{L}^{2} / 2 \rho^{2}+\rho^{2} / 8-\left(\rho^{2}+z^{2}\right)^{-1 / 2}$, for $\rho_{0}=1.45 \quad(\hat{L} \sim 1.5985)$. The contour lines are at reduced energies [Eq. (2.8)] $f=0.05$, $0.10,0.15,0.20,0.25,0.30,0.35$, and 0.40 .

$$
\begin{aligned}
& d \rho / d t=P_{\rho}, \quad d z / d t=P_{z}, \\
& d P_{\rho} / d t=-\rho /\left(\rho^{2}+z^{2}\right)^{3 / 2}-\frac{1}{4} \rho+\hat{L}^{2} / \rho^{3}, \\
& d P_{z} / d t=-z /\left(\rho^{2}+z^{2}\right)^{3 / 2} .
\end{aligned}
$$

The system can be most easily discussed if a number of parameters are defined and its general nature described. The potential energy has a minimum, denoted $V_{0}$, at $z=0, \rho=\rho_{0}$ :

$$
V_{0}=\rho_{0}^{-1}\left(\rho_{0}^{3} / 4-\frac{1}{2}\right) ;
$$

the minimum in $\rho$ is related to $\hat{L}$ by

$$
\hat{L}^{2}=\rho_{0}\left(\rho_{0}^{3} / 4+1\right) \text {. }
$$

The potential energy has the value of the classical escape energy, $V_{s}=\hat{L} / 2$, at the saddle point $z=\infty, \rho_{s}=(2 \hat{L})^{1 / 2}$. We define the reduced energy $f$ to measure the energy in this range:

$$
f=\left(E-V_{0}\right) /\left(V_{s}-V_{0}\right),
$$

where $E$ is the total energy of the system, $f=0$ is the bottom of the well, and $f=1$ is the escape energy. At $z=0$, we denote the minimum and maximum values of $\rho$ allowed by energy conservation as $\rho_{\min }$ and $\rho_{\max }$, respectively.

The Taylor expansion of the potential energy about its minimum plays an important role in the interpretation of the phase-space structure:

$$
\begin{aligned}
V(\rho, z)= & V_{0}+\frac{1}{2} k_{\rho}\left(\rho-\rho_{0}\right)^{2}+\frac{1}{2} k_{z} z^{2} \\
& +\frac{1}{2} c\left(\rho-\rho_{0}\right)^{3}+\frac{1}{2} d\left(\rho-\rho_{0}\right) z^{2}+\ldots,
\end{aligned}
$$

with 


$$
\begin{aligned}
& k_{\rho}=1+1 / \rho_{0}^{3}, \quad k_{z}=1 / \rho_{0}^{3}, \\
& c=-\frac{12 \hat{L}^{2}}{\rho_{0}^{5}}+\frac{6}{\rho_{0}^{4}}=-\frac{3}{\rho_{0}}\left(1+\frac{2}{\rho_{0}^{3}}\right), \\
& d=-\frac{3}{\rho_{0}^{4}} .
\end{aligned}
$$

If the cubic terms are neglected, then the $\rho$ and $z$ motions are independent harmonic oscillations about the equilibrium point, with frequencies (recall that scaling gives an effective mass of 1 )

$$
\begin{aligned}
& \omega_{\rho}^{0}=\left(k_{\rho}\right)^{1 / 2}, \\
& \omega_{z}^{0}=\left(k_{z}\right)^{1 / 2} .
\end{aligned}
$$

It is found that the transition regime occurs near that value of $\hat{L}$ (call it $\hat{L}_{T}$ ) such that $\omega_{\rho}^{0}=2 \omega_{z}^{0}$. Using Eq. (2.10), this resonance condition occurs when $k_{\rho}-4 k_{z}=0$, or

$$
1+\frac{1}{\rho_{0}^{3}}=\frac{4}{\rho_{0}^{3}} .
$$

The equation giving $\rho_{0}$ as a function of $\hat{L}$ was already given in Eq. (2.7), and these two equations together are easily solved for $\rho_{0}$ and $\hat{L}$,

$$
\begin{aligned}
& \rho_{0}^{T}=3^{1 / 3}=1.442 \ldots, \\
& \hat{L}_{T}=\left(\frac{7}{4} \rho_{0}^{T}\right)^{1 / 2}=\left(\frac{7}{4} \times 3^{1 / 3}\right)^{1 / 2}=1.589 \ldots .
\end{aligned}
$$

Central to our study is the fact that the Hamiltonian contains a parameter $(\hat{L})$; we examine the change of trajectories and eigenfunctions as this parameter changes. In many other studies, all parameters in the Hamiltonian were held fixed, and trajectories were studied only as a function of the energy. The complete picture does not emerge that way. Also central to our study is the fact that as our parameter varies, the zeroth-order frequency ratio $\omega_{\rho}^{0} / \omega_{z}^{0}$ also varies, and we can tune the Hamiltonian through the $2: 1$ resonance. This is different from the situation in Ref. 3(a), and it may account for the different conclusions we obtain.

\section{QUANTUM EIGENFUNCTIONS AND EIGENVALUES}

\section{A. Hamiltonian}

Using the same scaled variables defined above (recall that carets are omitted), the quantum Hamiltonian for this system is

$$
H_{Q}=-\frac{\hat{\hbar}^{2}}{2} \nabla^{2}+\frac{\hat{L}^{2}}{2 \rho^{2}}-\left(\rho^{2}+z^{2}\right)^{-1 / 2}+\rho^{2} / 8 .
$$

Here $\nabla^{2}$ is the Laplacian for the two reduced variables $(\rho, z)$,

$$
\nabla^{2}=d^{2} / d \rho^{2}+(1 / \rho) d / d \rho+d^{2} / d z^{2},
$$

and $\hat{\hbar}$ is the reduced value of $\hbar, \hat{\hbar}=\hbar / \alpha \beta$.

The value of $\hat{\hbar}$ determines the density of the quantum states. To examine the relationship between quantum states and classical trajectories, we need to choose $\hat{\hbar}$ such that about 20 quantum states exist in the transition regime. The value chosen was $\hat{\hbar}=\frac{1}{50}$, or equivalently, $\alpha \beta=50$ a.u. (This means that we study states with $L_{z} \approx 80$ in a magnetic field of about $1.88 \mathrm{~T}$.)

With the substitution

$$
\psi(\rho, z)=\rho^{-1 / 2} \Phi(\rho, z),
$$

the equation for $\Phi(\rho, z)$ is

$$
\begin{aligned}
& {\left[-\frac{\hat{\hbar}^{2}}{2}\left[\frac{d^{2}}{d \rho^{2}}+\frac{d^{2}}{d z^{2}}\right]+L^{\prime 2} / 2 \rho^{2}\right.} \\
& \left.-\left(\rho^{2}+z^{2}\right)^{-1 / 2}+\rho^{2} / 8-E\right] \Phi(\rho, z)=0,
\end{aligned}
$$

where $L^{\prime 2}=\hat{L}^{2}-\hat{\hbar}^{2} / 4$. The wave function must vanish as $\rho \rightarrow 0$, and it has a branch point there.

Eigenfunctions are calculated by expansion in a basis. The most convenient basis is the set of harmonicoscillator functions centered at the minimum of the potential energy, $z=0, \rho=\rho_{0}$. These basis functions do not have quite the right boundary conditions; they go to zero as $\rho \rightarrow-\infty$ instead of as $\rho \rightarrow 0$. However, the error introduced by this is small, because the origin is far into a classically forbidden region. (For example, the value of the lowest harmonic-oscillator function is about $10^{-26}$ at the origin.)

Parameters in the harmonic-oscillator basis are $k_{\rho}$ and $k_{z}$, specified in Eq. (2.10). The zeroth-order Hamiltonian is separable and has as its eigenfunctions products of harmonic-oscillator functions, with zeroth-order energies

$$
E_{n_{\rho}, n_{z}}^{0}-V_{0}=\left(n_{\rho}+\frac{1}{2}\right) \hat{\hbar} \omega_{\rho}^{0}+\left(n_{z}+\frac{1}{2}\right) \hat{\hbar} \omega_{z}^{0} .
$$

The full quantum solution, with eigenvalue $E_{n_{\rho}, n_{z}}$, is obtained variationally with basis sets of 72 functions, 12 in $\rho$ and 6 in $z$ (the parity symmetry in $z$ is used to reduce the size of the matrix); the energy of the highest reported levels is converged to within $0.1 \%$ under these conditions. Both variational and zeroth-order eigenvalues are converted to reduced energies according to Eq. (2.8).

\section{B. Energies, wave functions, and correlations}

The variationally computed energies for a number of $L^{\prime}$ values are listed in Table I and plotted in Fig. 2. Well away from $L_{T}^{\prime} \equiv\left(\hat{L}_{T}^{2}-\hat{\hbar}^{2} / 4\right)^{1 / 2}$, the assignment of quantum numbers is unambiguous, so in the table the entries at the smallest and largest $L^{\prime}$ values are identified using an $\left(n_{\rho}, n_{z}\right)$ notation. In the figure the variationally computed eigenvalues $f_{k}$ are given by the symbols, while the lines represent the zeroth-order energies, $f^{0}\left(n_{\rho}, n_{z}\right)$, which can be unambiguously labeled with quantum numbers throughout the diagram. Both the zeroth-order and variational results show the degeneracy pattern expected near a 2:1 resonance, with the variational results having an avoided crossing. The zeroth-order results cross and provide only a qualitative description of the energy behavior near the resonance.

It is well known that eigenstates exchange character upon passage through an avoided crossing. For example, 
TABLE I. Quantum eigenvalues $(\hat{\hbar}=1 / 50$; entries are $100 f)$.

\begin{tabular}{rrrrrrrrr}
\hline \hline$n_{\rho}, n_{z}$ & 1.3 & 1.4 & 1.5 & 1.6 & 1.7 & 1.8 & 1.9 \\
0,0 & & & & & & & \\
0,1 & 2.82 & 2.79 & 2.78 & 2.78 & 2.79 & 2.80 & 2.83 \\
1,0 & 4.89 & 4.58 & 4.65 & 4.58 & 4.58 & 4.49 & 4.46 \\
0,2 & 6.27 & 6.29 & 6.31 & 6.27 & 6.19 & 6.11 & 6.05 \\
1,1 & 6.92 & 6.70 & 6.57 & 6.56 & 6.62 & 6.71 & 0,1 \\
0,3 & 8.25 & 8.15 & 8.04 & 7.92 & 7.80 & 7.69 & 7.59 \\
2,0 & 8.93 & 8.64 & 8.47 & 8.40 & 8.39 & 8.42 & 8.47 \\
1,2 & 9.71 & 9.77 & 9.69 & 9.53 & 9.37 & 9.22 & 1,0 \\
0,4 & 10.21 & 10.06 & 10.04 & 10.06 & 10.06 & 10.05 & 10.10 \\
\hline \hline
\end{tabular}

the first two excited states of odd parity are predominantly the $(1,1),(0,3)$ pair of states, which are degenerate at $L^{\prime}=L_{T}^{\prime}$ in zeroth order. For $L^{\prime} \ll L_{T}^{\prime}$, the state of lower energy has quantum numbers $(1,1)$, while for $L^{\prime} \gg L_{T}^{\prime}$, it has quantum numbers $(0,3)$. How does this change of character occur?

Contour plots of the wave functions for the same excited states of odd parity are shown for a sequence of $L^{\prime}$ values in Fig. 3; Figs. 3(a)-3(d) display the first excited state for $L^{\prime}=1.3,1.5,1.6$, and 1.7 , respectively, and Figs. 3(e) $-3(\mathrm{~h})$ display the second excited state. The heavy line in the figure demarks an energetically allowed region; the fine outline is discussed below. Figure 3(a) shows a nodal structure consistent with the $(1,1)$ assignment of quantum numbers, while Fig. 3(d) is clearly a $(0,3)$ state; the sequence shows that the transmogrification from the $(1,1)$ to the $(0,3)$ pattern in this state occurs by an opening up of the wave function on the right. The other member of the degenerate pair shows a similar change, but from a $(0,3)$ structure to a $(1,1)$ structure, by closing on the left. For the intermediate $L^{\prime}=1.5$ value, the assignment of a set of quantum numbers would be difficult; the states are highly mixed.

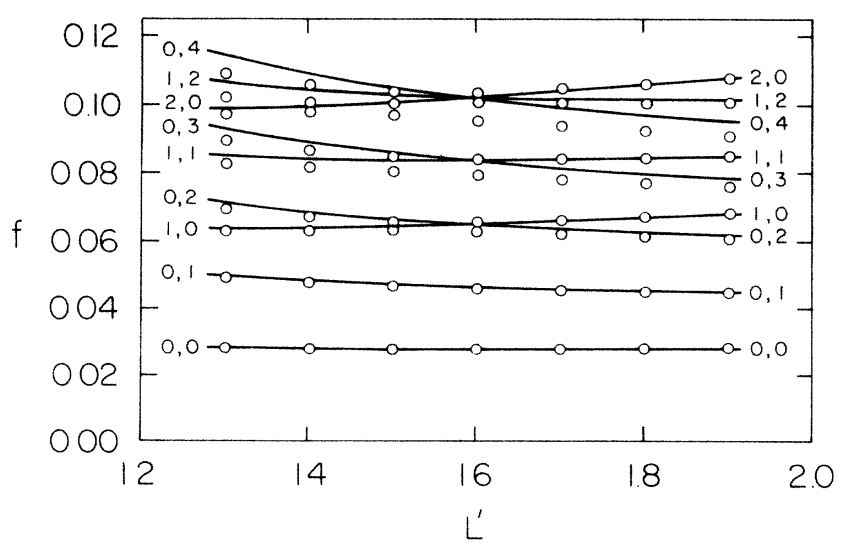

FIG. 2. Quantum energies $\varepsilon$ as functions of the parameter $L$, for $\alpha \beta=50$, expressed as reduced energies $f$. The lines give the zeroth-order energies; the symbols, variational results. The zeroth-order results are labeled with their quantum numbers.

\section{PHASE-SPACE STRUCTURE}

We now examine classical trajectories, and study their relationship to the quantum eigenstates. Since we seek such a relationship, we must use a classical Hamiltonian that corresponds most closely to the quantum Hamiltonian (3.4) and the boundary conditions actually satisfied by the computed wave functions. That classical Hamiltonian is (in scaled variables)

$H=\frac{1}{2}\left(P_{\rho}^{2}+P_{z}^{2}\right)+L^{\prime 2} / 2 \rho^{2}-\left(\rho^{2}+z^{2}\right)^{-1 / 2}+\rho^{2} / 8$.

It differs from the one in Eq. (2.3) in the replacement of $\hat{L}^{2}$ by $L^{\prime 2}=\hat{L}^{2}-\hat{\hbar}^{2} / 4$. (Appendix A contains a discussion of this point. ${ }^{7}$ )

In Ref. 1(b), we showed that two families of regular trajectories exist in the transition regime. One family correlates to elliptical trajectories in the low-field limit, and so is called "E;" the other correlates to helical trajectories in the high-field limit, and is termed "H." Using $L^{\prime}=1.51$ as representative of the transition regime, typical trajectories of each type are shown in Fig. 4.

Composite Poincaré surfaces of section give a picture of the phase-space structure of the trajectories. For this system, surfaces of section are obtained by recording $P_{\rho}$ and $\rho$ each time a trajectory reaches $z=0$ with $P_{z}>0$. In this case, $\rho$ is the rapidly oscillating variable and $z$ is the slowly oscillating one. A composite of surfaces of section at a fixed energy $f$, made up from a number of trajectories of each family, is shown in Fig. 5. For each family there is one periodic trajectory which crosses the $z=0$ plane at a single point; the locations of these periodic trajectories, designated $\rho_{\mathrm{H}}$ and $\rho_{\mathrm{E}}$, respectively, occur at $P_{\rho}=0$ with $\rho_{\mathrm{H}}<\rho_{\mathrm{E}}$.

In the transition regime, the composite surfaces of section can be characterized as follows. If we fix $f$, and let $L^{\prime}$ be significantly less than 1.5 , only E-type trajectories appear. If $L^{\prime}$ is increased, then at some critical value a small set of H-type trajectories appears, and occupies a little space at small $\rho$ on the $\rho, P_{\rho}$ plane. As we continue to increase $L^{\prime}$, the separatrix between $\mathrm{E}$ and $\mathrm{H}$ trajectories moves toward larger $\rho$ and the fraction of phase space occupied by $\mathrm{H}$-type trajectories grows. Finally, at a second critical value of $L^{\prime}$, the $E$ trajectories disappear. The band in $L^{\prime}$ space in which both types coexist is smaller at smaller $f$. The bifurcation boundary is the 

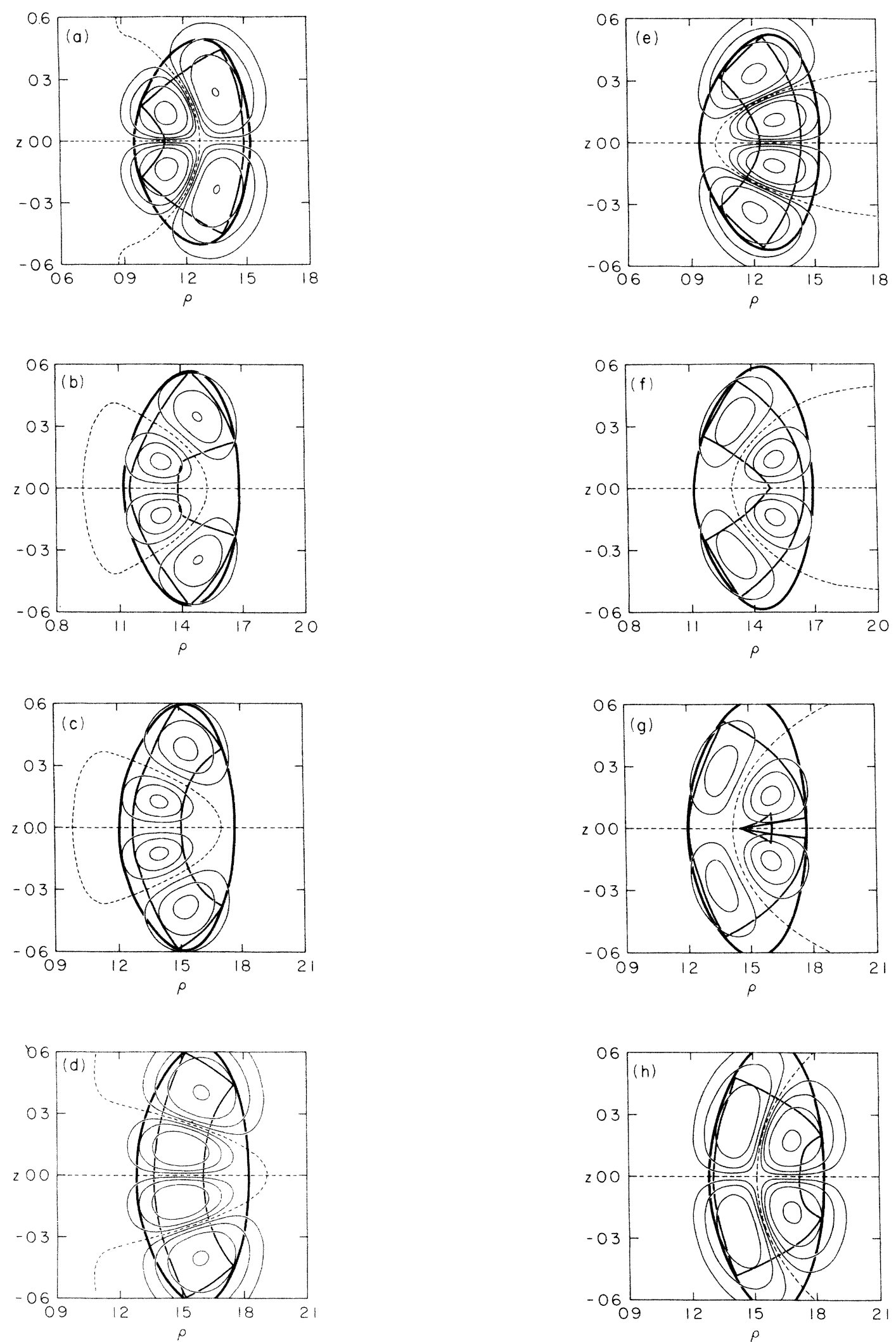

FIG. 3. Contour plots of variationally computed wave functions for $\alpha \beta=50$. The first excited state of odd parity [corresponding to state $(1,1)$ at small $\hat{L}$ is shown in (a)-(d); the next excited state of odd parity is shown in (e)-(h). $\hat{L}=1.3$ in (a) and (e); $\hat{L}=1.5$ in (b) and $(\mathrm{f}) ; \hat{L}=1.6$ in (c) and $(\mathrm{g}) . \hat{L}=1.7$ in (d) and $(\mathrm{h})$. The energies of these states are given in Table I. Superimposed on each is the caustic of the semiclassical eigentrajectory; the heavy line is the potential contour corresponding to the semiclassical eigenenergy (Table II). 
curve $f_{\text {bif }}\left(L^{\prime}\right)$ in the $f-L^{\prime}$ plane at which a family of trajectories first appears as the energy increases.

The bifurcation boundary is located by the following procedure. A value of $L^{\prime}$ is selected, and $\rho_{\mathbf{H}}(f)$ and $\rho_{\mathbf{E}}(f)$ are generated for increasing values of the reduced energy $f$. Since a trajectory needs to be integrated for only one loop to find a periodic trajectory, and since only a few sets of initial values $\left(f, z^{I}=0, P_{\rho}^{I}=0, \rho^{I}\right)$ generally suffice to find the periodic trajectory, this procedure requires little computer time. A plot of $\rho_{\mathbf{H}}(f ; L)$ and $\rho_{\mathbf{E}}(f ; L)$ versus $f$ will then show that for $f$ below a critical value, of the periodic trajectories does not occur; this value is identified as $f_{\text {bif }}$. An example is shown in Fig. 6.

Using this procedure, a given set of computations can identify the bifurcation boundary, shown in Fig. 7, to within a finite precision. The size of the points in the figure represents our estimate of the uncertainty in the boundary due to the finite resolution available in our calculations.

We see from Figs. 4 and 5 that the separatrix marks a discontinuous change in the character of trajectories. Nonetheless, there is a way to assign a correlation to trajectories on opposite sides of the separatrix. Consider the trajectory with initial conditions $\left(f, z^{I}=0, P_{\rho}^{I}=0\right.$, $\rho^{I}=\rho_{0}$ ). This trajectory starts with $\rho$ at the bottom of the well, with initial velocity in the $z$ direction only. As $L^{\prime}$ increases, the separatrix passes through $\rho_{0}$, and the character of the trajectory changes from type $E$ to type $H$. The sequence of trajectories shown in Fig. 8 demonstrates this behavior. As the separatrix approaches $\rho_{0}$ the trajectory expands to fill nearly all of the available configuration space, except for a narrow slit on the left at $z=0$. Upon passage through the separatrix, the shape of the trajectory is very similar except that the slit at $z=0$ now extends to the right.

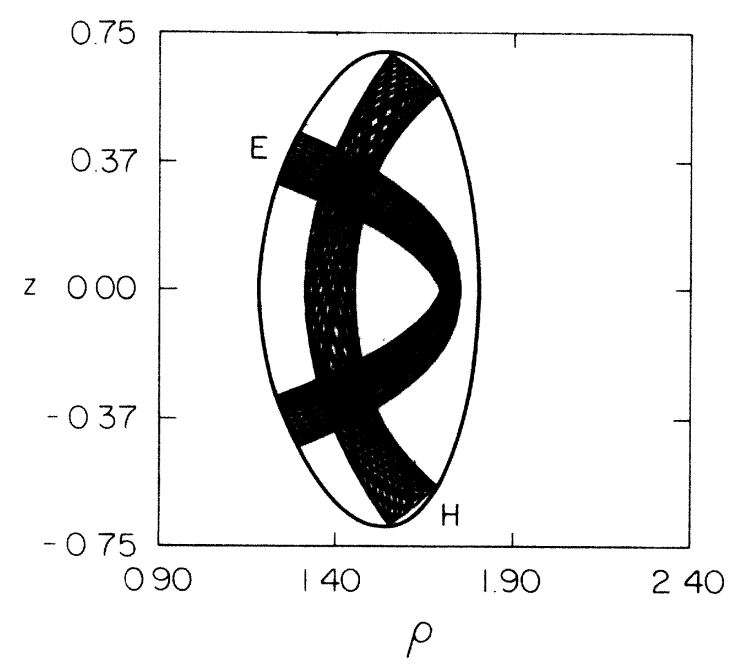

FIG. 4. Two trajectories in the transition regime at $\hat{L}=1.60$. Type $\mathrm{E}$ are related to elliptical trajectories; type $\mathbf{H}$, to helical trajectories. Initial conditions are $P_{\rho}=0, z=0, f=0.1$, and type E: $\rho=1.75$; type $\mathrm{H}: \rho=1.31$.
Thus, trajectories very close to the separatrix occupy very much the same region in configuration space. We should emphasize, however, that the phase space occupied by the trajectories is very different, as the surface of section shows, and that the classical actions generated by the trajectories are usually very different. Note that the trajectories close to the separatrix exhibit the behavior described by Herzberg quoted above. To be precise, such trajectories begin with most of the energy in $\rho$ motion, and a tiny amplitude of $z$ motion; through many cycles of $\rho$ motion, the amplitude of $z$ motion increases, first very gradually, then more rapidly; for less than one cycle, most of the energy is in $z$ motion, but it is quickly transferred back to $\rho$ motion, and the $z$ amplitude decreases again to a small value. Then the whole process repeats. However, we should emphasize that this description applies only to trajectories close to the separatrix; as Fig. 8 shows, most of the trajectories would not be described this way.

In a later section we shall show that Floquet analysis and reduction to Mathieu's equation provide an interpretation of the bifurcation of the family of trajectories. First, however, we compute trajectories that are correlated with eigenstates.

\section{SEMICLASSICAL EIGENVALUES, EIGENTRAJECTORIES, AND CORRELATIONS}

\section{A. Method of calculation}

For regular trajectories, allowed semiclassical energy levels can be found by quantizing classical actions. We have determined semiclassical eigenvalues for values of the parameter $L^{\prime}$ at intervals between 1.30 and 1.90 , using the surface-of-section (SOS) semiclassical method. ${ }^{5}$ The first step of this procedure involves the integration ${ }^{8}$ of the classical equations of motion. Initial conditions can be taken to be $z^{I}=0, \rho^{I}=$ arbitrary, $P_{\rho}^{I}=0, P_{z}^{I}$ $=\left\{2\left[E_{s}-V\left(\rho^{I}, z^{I}\right)\right]\right\}^{1 / 2}$. (Every trajectory passes through $z=0$ at least once, and the surfaces of section show that every torus includes a point where $z=0$ and $P_{\rho}=0$.) Together with the trajectory integration, we simultaneously integrate an additional equation in order to calculate the classical phase

$$
d S / d t=P_{z} d z / d t+P_{\rho} d \rho / d t
$$

The next step is to calculate the classical action for the $\rho$ motion $I_{\rho}$ as the area of the Poincaré surface at $z=0$, $P_{z}>0$ (see Fig. 5),

$$
I_{\rho}=\oint P_{\rho} d \rho
$$

To improve the numerical precision, this integral is performed using circular polar coordinates suitably centered in the $\left(P_{\rho}, \rho\right)$ plane. ${ }^{9}$ The total action $I$ is found by the loop-closure method. ${ }^{5(\mathrm{c})}$. The total action is given by the difference between any value of $S$ on the SOS and its value upon its next return to the SOS, corrected by the 
closure integral $\int P_{\rho} d \rho$ between the two points. The relationship

$$
I=N_{c} I_{\rho}+I_{z}
$$

allows calculation of $I_{z}$ once the integer $N_{c}$ is determined. The parameter $N_{c}$ counts the number of loops in $\rho$ made by the trajectory in completing one loop in $z$ from the SOS and back. From the shape of the trajectory, or the fact that we are near the 2:1 resonance, $N_{c}$ usually has the value 2 . However, for some trajectories at large $L$, as we move away from the 2:1 resonance zone, $N_{c}$ can be 3 . We only observed this for helical trajectories, for which the sign of the momentum upon return to the SOS unam-
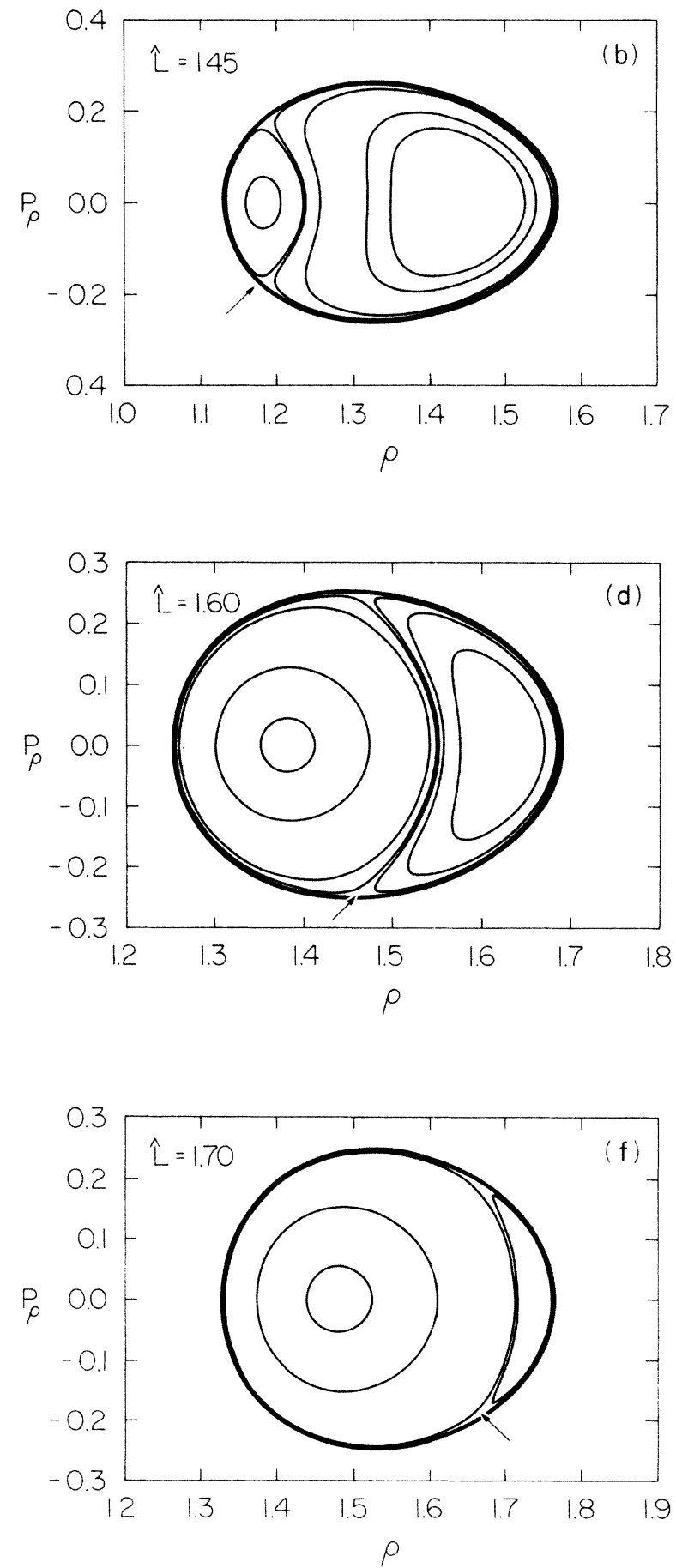

FIG. 5. A composite of surfaces of section in the transition regime with $z=0, P_{z}>0$. The heavy outer lines enclose the energyallowed region. The ellipses at smaller $\rho$ are type-H surfaces of section; at larger $\rho$, type E. The arrow indicates the position of the bifurcation boundary, according to Appendix B. (a) $L=1.40$, (b) $L=1.45$, (c) $L=1.55$, (d) $L=1.60$, (e) $L=1.65$, and (f) $L=1.70$. 


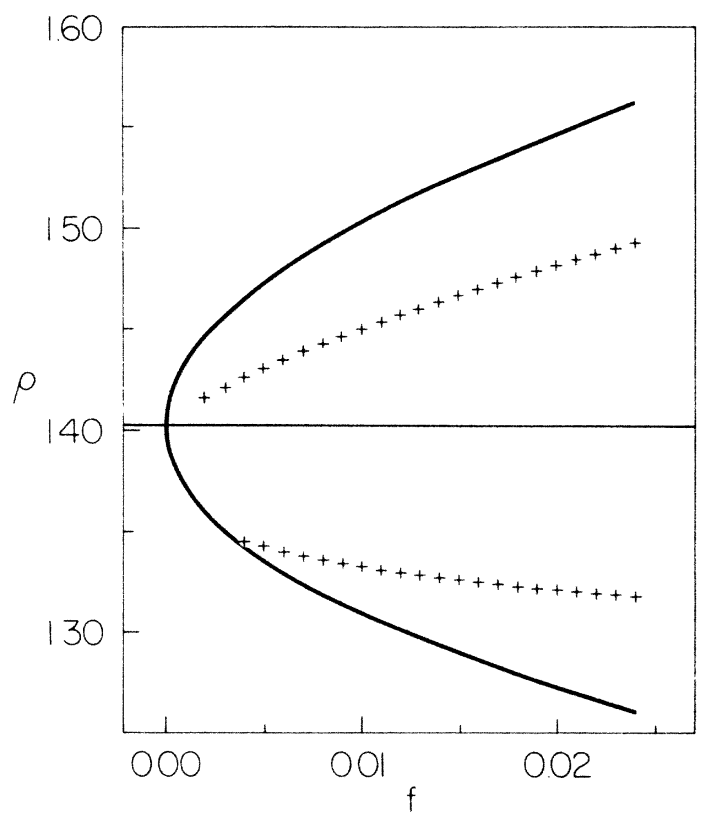

FIG. 6. A plot of the position at which the periodic trajectory crosses the $z=0$ plane as a function of reduced energy $f$ for $L^{\prime}=1.54$. The heavy curve locates $\rho_{\max }$ and $\rho_{\min }$, while the middle line is the constant $\rho=\rho_{0}$. The upper set of symbols gives the positions $\rho_{\mathrm{E}}(f)$, the lower gives $\rho_{\mathrm{H}}(f) . f_{\text {bif }}\left(L^{\prime}\right)$ is found by estimating where the curve $\rho_{\mathrm{E}}(f)$ [or $\rho_{\mathrm{H}}(f)$ ] intersects the $\rho_{\max }$ (or $\rho_{\min }$ ) boundary.

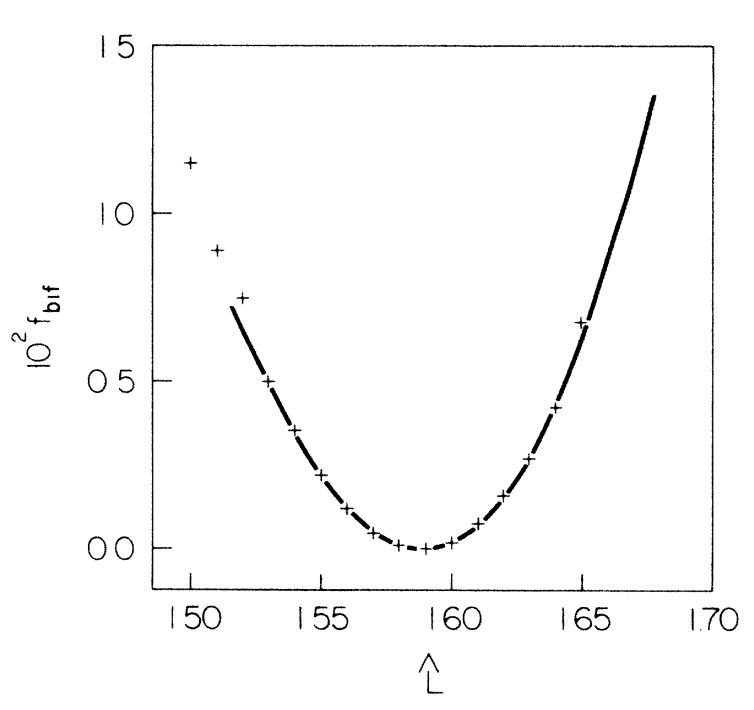

FIG. 7. A plot of the bifurcation boundary $f_{\text {bif }}(\hat{L})$ as a function of $\hat{L}$. The symbols give the positions as found from plots such as Fig. 6 . The solid curve is $f_{\text {bif }}(\hat{L})$, as determined by the Floquet-Mathieu analysis. biguously determines the value of $N_{c}$. For example, if the trajectory starts at the minimum possible $\rho$ value, on the left-hand caustic, then upon return to the SOS if $P_{\rho}$ is positive, only two loops in $\rho$ have occurred. If $P_{\rho}$ is negative, then the right-hand caustic has been touched again in the process of beginning the third loop, and $N_{c}$ is 3 .

This procedure is used to find actions at a number of different initial conditions for a variety of $f$ values, usually $f=0.05,0.10,0.15$, and 0.20 . Each trajectory is identified as $\mathrm{E}$ or $\mathrm{H}$, and trajectories starting near $\rho_{\text {min }}$ and near $\rho_{\max }$ are included so as to obtain the largest possible $I_{\rho}$ for both types $\mathrm{E}$ and $\mathrm{H}$.

In Fig. 9, we show a three-dimensional plot of the surfaces $f\left(n_{\rho}, n_{z}\right)$ regarding $n_{\rho}=\left(I_{\rho} / 2 \pi \hbar-\frac{1}{2}\right)$ and $n_{z}=\left(I_{z} / 2 \pi \hat{\hbar}-\frac{1}{2}\right)$ as continuous variables. Notice that only a portion of the plane is occupied: for each given $n_{\rho}$ there is a minimum value of $n_{z}$ that is dynamically allowed. The point at $n_{\rho}=-\frac{1}{2}, n_{z}=-\frac{1}{2}$ in the figure corresponds to both actions being zero and $f=0$. It occurs for helical states for all $L^{\prime}>L_{T}^{\prime}$, and for elliptical states for $L^{\prime}<L_{T}^{\prime}$. For each value of $L^{\prime} \neq L_{T}^{\prime}$, only one type of trajectory is found at very small $f$, and a second type occurs when $f$ increases above the critical value, $f_{\text {bif }}\left(L^{\prime}\right)$. At its first appearance, that new type of trajectory has $n_{\rho}=-\frac{1}{2}$ and $n_{z}$ equal to some value greater than $-\frac{1}{2}$. (The area on the $z=0$ surface of section is vanishingly small, but the amplitude and action associated with the motion in $z$ are not small.) In practice, we notice that for energies $f$ not too far from the bifurcation boundary, the function $I / f$ is a linear function of $f$; we exploit this to extrapolate the value of $I$ at $f=f_{\text {bif }}$ providing additional data for an interpolation.

The conditions which identify the "eigentrajectories," the trajectories for which the action variables obey the Einstein-Brillouin-Keller quantization conditions, are $^{3}$

$$
\begin{aligned}
& I_{z}=\left(n_{z}+1 / 2\right) 2 \pi \hat{\hbar}, \\
& I_{\rho}=\left(n_{\rho}+1 / 2\right) 2 \pi \hat{\hbar} .
\end{aligned}
$$

A systematic two-dimensional interpolation procedure was implemented to accomplish this. ${ }^{10}$ Numerical calculations give a set of values of $I_{\rho}$ and $I_{z}$ for selected values of $f$ and $\rho^{I}$. A numerical interpolation effectively generates the function $f=f\left(I_{\rho}, I_{z}\right)$; setting $I_{\rho}$ and $I_{z}$ to the quantized values produces the semiclassical eigenvalues directly.

A check on the interpolation procedure was also performed. A second numerical interpolation generates the function $\rho^{I}=\rho^{I}\left(I_{\rho}, I_{z}\right)$; the same quantized values of the actions then generate the remaining initial condition needed to integrate a trajectory and evaluate the actions directly. If both interpolations are accurate, the directly determined values are the quantized values. We used this check whenever we were uncertain of the accuracy of the interpolation procedure.

Since the surfaces $f\left(n_{\rho}, n_{z}\right)$ shown in Fig. 9 are nearly planar, the interpolation procedure is very accurate. For $\alpha \beta=50$, results are tabulated in Table II and plotted in Fig. 10, with the open circles denoting helical states and 

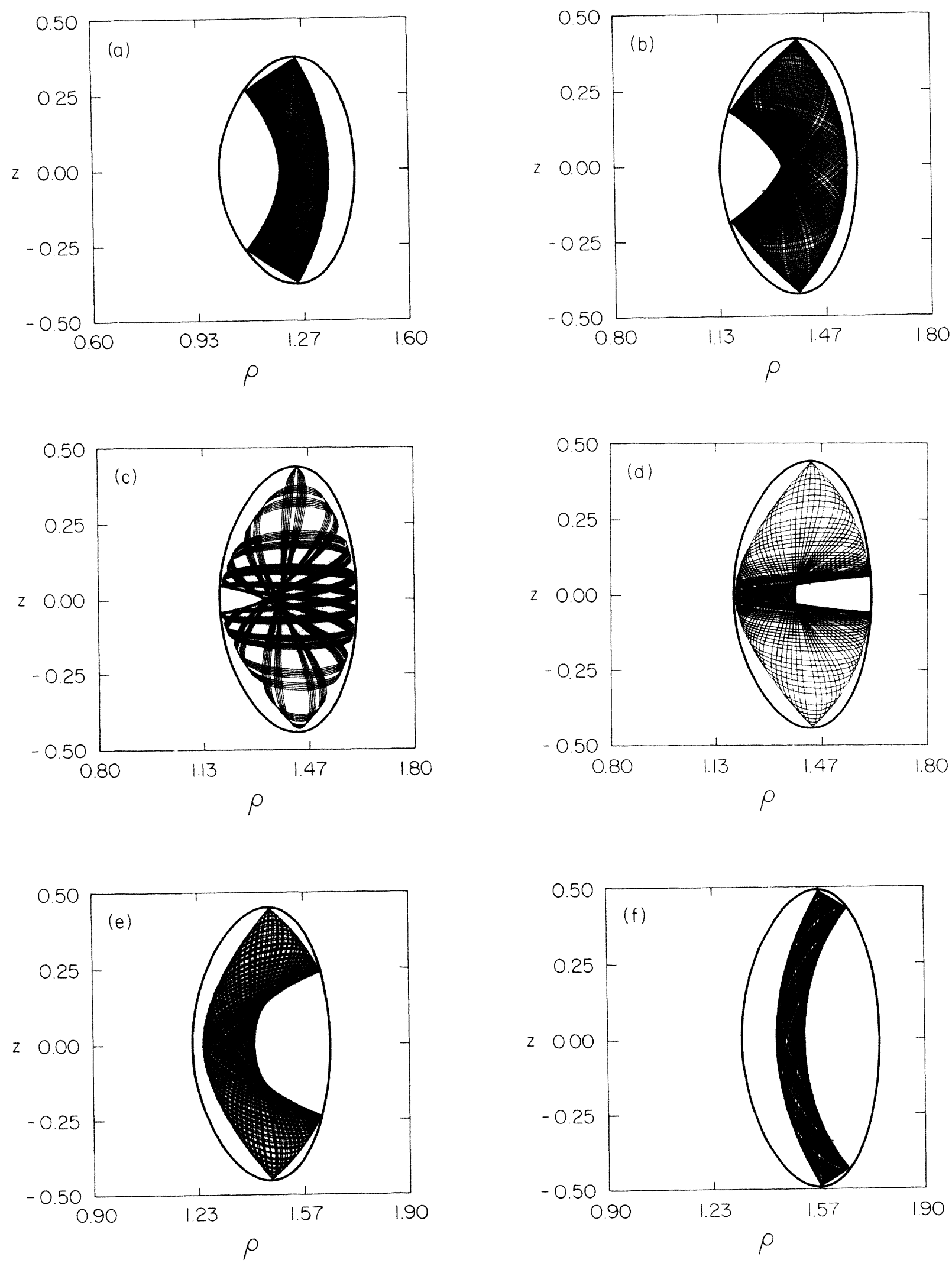

FIG. 8. A sequence of trajectories with initial conditions $f=0.05, z^{I}=0, P_{\rho}^{I}=0, \rho^{I}=\rho_{0}$, for increasing $\rho_{0}$, corresponding to increasing $L$. The sequence shows the correlation between trajectories belonging to different families. (a) $\hat{L}=1.3$; (b) $\hat{L}=1.45$; (c) $\hat{L}=1.510$; (d) $\hat{L}=1.515$; (e) $\hat{L}=1.55$; and (f) $\hat{L}=1.70$. 

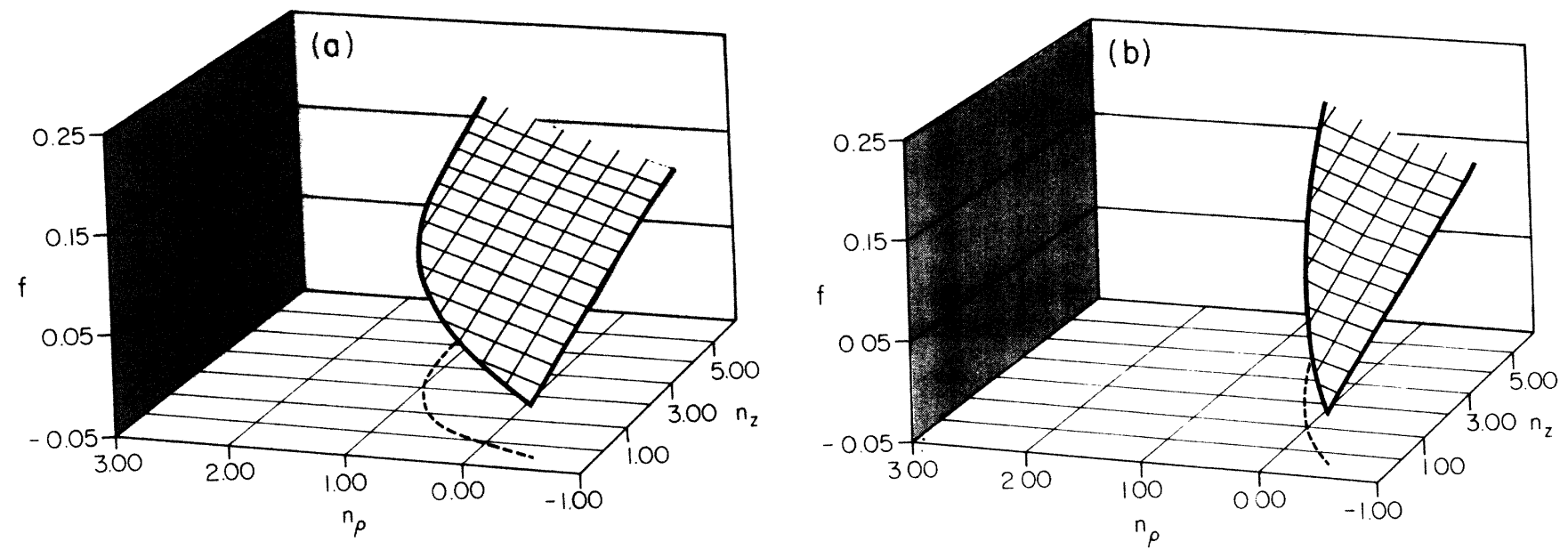

FIG. 9. (a) The surface $f_{\mathrm{H}}\left(n_{\rho}, n_{z}\right)$ in which the "quantum numbers" are regarded as continuous variables. The values of the surface at integer grid intersections are the semiclassical eigenvalues. The dotted line in the $\left(n_{\rho}, n_{z}\right)$ plane locates the minimum value of $n_{z}$ at each given value of $n_{\rho}$. (b) as in (a), but for $f_{\mathrm{E}}\left(n_{\rho}, n_{z}\right)$.

solid circles denoting elliptical states. The accuracy of the eigenvalues is on the order of $1 \%$.

\section{B. Semiclassical correlations}

\section{Energy eigenvalues}

Semiclassical eigenvalues are compared with quantum eigenvalues in Fig. 10. The most important fact about the semiclassical eigenvalues is quite evident from this figure. They closely follow the quantum eigenvalues, and in particular, they also show avoidance of crossing. This result would not be surprising in the least, were it not for the fact that the opposite result was obtained in Ref. 3(a); there, the semiclassical eigenvalues crossed. We will return to this point in our conclusions.

Let us now examine the relationship between quantum and semiclassical states in more detail. For small $L^{\prime}$, each semiclassical state considered corresponds to an Etype trajectory, and at large $L^{\prime}$ to an $H$-type trajectory. For each state, as $L^{\prime}$ increases there is some value of $L$ at which the quantized E-type trajectory disappears, and there is a value of $L^{\prime}$ at which the corresponding quantized H-type trajectory appears. Interesting little discrepancies between semiclassical and quantum results arise from the fact that the replacement of quantized E-

TABLE II. Semiclassical eigenvalues $(\hat{\hbar}=1 / 50$; entries are $100 f)$.

\begin{tabular}{|c|c|c|c|c|c|c|c|c|}
\hline$n_{\rho}, n_{z} L^{\prime}$ & 1.3 & 1.4 & 1.5 & 1.6 & 1.7 & 1.8 & 1.9 & $n_{\rho}, n_{z}$ \\
\hline 0,0 & 2.81 & 2.78 & 2.75 & & & & & \\
\hline & & & & 2.80 & 2.81 & 2.81 & 2.83 & 0,0 \\
\hline 0,1 & 4.88 & 4.73 & 4.64 & & & & & \\
\hline 1,0 & 625 & 6.27 & & 4.56 & 4.53 & 4.49 & 4.47 & 0,1 \\
\hline & & & & & 6.64 & 6.73 & 6.83 & 1,0 \\
\hline 0,2 & 6.91 & 6.69 & 6.59 & 6.62 & & & & \\
\hline 1,1 & 8.26 & 8.14 & 6.33 & 6.27 & 6.18 & 6.13 & 6.10 & 0,2 \\
\hline 0,3 & 80 & & 840 & $\begin{array}{l}8.35 \\
8.49\end{array}$ & 8.38 & 8.42 & 8.47 & 1,1 \\
\hline 0,3 & 8.92 & 8.64 & $\begin{array}{l}8.49 \\
8.03\end{array}$ & $\begin{array}{l}0.47 \\
7.92\end{array}$ & 7.80 & 7.68 & 7.59 & 0,3 \\
\hline 2,0 & 9.66 & & & & & & & \\
\hline 1,2 & 10.26 & 10.10 & 10.17 & & & 10.64 & 10.84 & 2,0 \\
\hline & & & & 10.06 & 10.03 & 10.01 & 10.10 & 1,2 \\
\hline 0,4 & 10.91 & 10.56 & 10.39 & 10.47 & & & & \\
\hline & & 9.76 & 9.68 & 9.53 & 9.39 & 9.23 & 9.09 & 0,4 \\
\hline
\end{tabular}




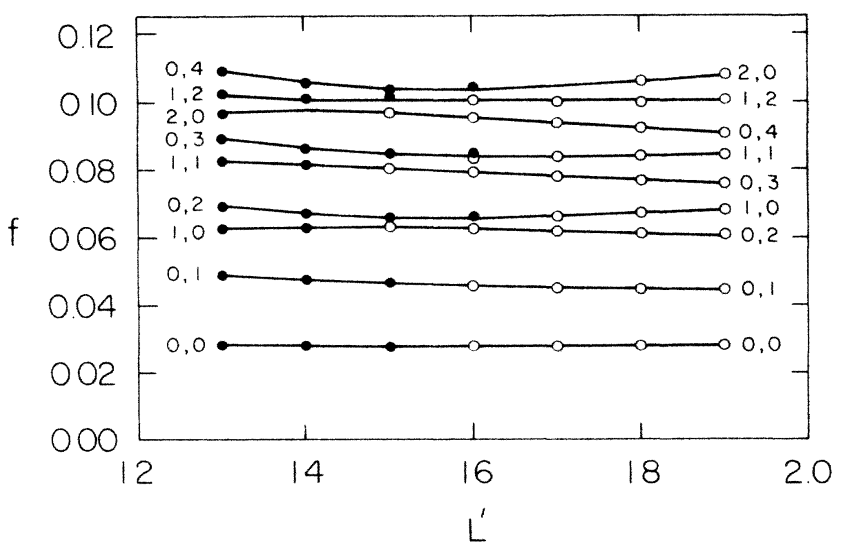

FIG. 10. Semiclassical energies $E_{n_{\rho_{z}}}$ as functions of the parameter $\hat{L}$ for $\alpha \beta=50$, expressed as reduced energies $f$. The solid circles denote E-type states, and the open circle denotes H-type states. The lines connect the variationally computed quantum results, shown also as the symbols in Fig. 2.

type eigentrajectories by $\mathrm{H}$-type eigentrajectories does not occur on a one-for-one basis.

As shown in Fig. 9, the surfaces $f_{E}\left(n_{\rho}, n_{z}\right)$ and $f_{H}\left(n_{\rho}, n_{z}\right)$ cover only a portion of the $\left(n_{\rho}, n_{z}\right)$ space, terminating at some maximum $n_{\rho}(f)$, and possibly starting at $n_{z}>-\frac{1}{2}$. This behavior occurs simply because the phase space is divided between the two classes of motion. The appearance of the separatrix restricts the maximum value of the area $I_{\rho}$ for either class to be less than the total classically accessible area of the $\left(\rho, P_{\rho}\right)$ plane. In addition, since $f_{\text {bif }}>0, I_{\rho}\left(f_{\text {bif }}\right)=0$ but $I_{z}\left(f_{\text {bif }}\right)>0$ for the class which is born at $f=f_{\text {bif }}$.

One consequence of this is missing eigentrajectories. At any $L^{\prime}$, we can find a value of $n_{\rho}$ larger than the maximum on the $f_{E}\left(n_{\rho}, n_{z}\right)$ surface for the value of $n_{z}$ desired; such a state cannot be represented at the primitive semiclassical level. The value of $n_{\rho}$ need not be large: For example, near $\hat{L}_{T}$ neither $\mathrm{E}$ nor $\mathrm{H}$ types of motion support a trajectory corresponding to the ground state. Specifically, for a small range of $L^{\prime}$ near $L^{\prime}=1.58$, there is no E-type or H-type trajectory having $n_{\rho}=0$ and $n_{z}=0$. (The semiclassical representation of this state requires "tunneling" or "coupling" between the E and $\mathrm{H}$ motions.)

A clear example of missing states is visible in Fig. 10. Consider that state which has quantum numbers $(2,0)$ at $L^{\prime}=1$.30. As $L^{\prime}$ increases, somewhere between $L^{\prime}=1.3$ and 1.4 the $(2,0)$ E-type eigentrajectory disappears (the separatrix annihilates it). Later, at $L^{\prime}$ between 1.7 and 1.8 , a $(2,0) \mathrm{H}$-type eigentrajectory appears. In the entire range between 1.4 and 1.7 , there is no $(2,0)$ state of either type. That by itself does not mean that semiclassical states are missing. Starting from large $L^{\prime}$, there is an $(0,4) \mathrm{H}$-type state; as $L^{\prime}$ decreases, it persists throughout most of the transition regime, until it finally disappears somewhere between $L^{\prime}=1.50$ and 1.40 . We see from the diagram that one of the quantum states correlates with the $(2,0)$ E trajectory for $L^{\prime} \leq 1.3$ and the same state correlates with the $(0,4) \mathrm{H}$ trajectory for $L^{\prime} \geq 1.5$. However, near $L^{\prime}=1.4$, neither of these eigentrajectories exists. Again a semiclassical description of the quantum state would have to involve superposition or tunneling.

A second consequence is the appearance of extraneous eigentrajectories. In Fig. 10, at $L^{\prime}=1.60$, the sixth energy level is marked with both symbols. For this energy a $(1,1) \mathrm{H}$ eigentrajectory and a $(0,3) \mathrm{E}$ eigentrajectory coexist; at smaller $L^{\prime}$ the former disappears, and at larger $L^{\prime}$ the latter disappears, but at this point, the present primitive semiclassical approximation gives an extra "state."

\section{Eigenfunctions and eigentrajectories}

An additional comparison between the semiclassical and quantum results is possible by considering the eigentrajectory. As stated earlier, by interpolating to find $\rho^{I}\left(n_{\rho}, n_{z}\right)$, we can obtain initial conditions for the eigentrajectory. These trajectories were computed and plotted. In Fig. 3 we show for each eigenfunction the outline of the corresponding eigentrajectory.

The excellent agreement between the quantum and semiclassical description of the position and structure of the state is evident from the figures. ${ }^{11}$ The semiclassical states are unambiguously labeled by quantum numbers; E-type states are used at $L^{\prime}=1.3$, and $\mathrm{H}$-type states at $L^{\prime}=1.6$ and 1.7. At 1.5 we used the states $(0,3) \mathrm{H}$ and $(0,3)$ E; they can be seen from Tables I and II to be in good agreement with the quantum eigenvalues, and from Fig. 3(b) and 3(f) to provide a good description for both wave functions. The figures show clearly that the change of character of the quantum states and of the eigentrajectories closely parallel each other.

\section{FLOQUET-MATHIEU ANALYSIS OF STABILITY AND BIFURCATIONS}

We now abandon quantum mechanics entirely to examine a different question. Can we obtain a simple model to predict and interpret the bifurcation of the classical family of trajectories? We shall show that the bifurcation is correlated with a change in the stability of the periodic orbit lying on the $\rho$ axis. (Here we adapt and extend the methods given in Ref. 5.)

\section{A. Floquet-Mathieu analysis}

According to Eq. (2.3) or Eq. (2.9), if we examine the potential energy as a function of $z$ at any fixed $\rho$, we find a quadratic minimum in $V(z ; \rho)$ along the $\rho$ axis $(z=0)$. It follows that at any energy, and for any value of $L^{\prime}$, there is a periodic orbit lying on this axis. Is this periodic orbit stable? That is, if we start a particle near the periodic orbit with a tiny $z$ displacement, will it stay close to the $\rho$ axis? Intuitively, one might guess that it is always stable. First, if $\rho$ were held fixed, then certainly the electron would undergo stable, approximately harmonic oscillations in $z$. Second, normal-mode analysis tells us 
that for small oscillations the $z$ and the $\rho$ motions are uncoupled, and the energies associated with $\rho$ motion and $z$ motion are separately conserved. Therefore a small oscillation in $z$ should stay small.

In fact, however, this conclusion fails near resonance. Away from resonance, when $L^{\prime}$ is not close to $L_{T}^{\prime}$ then at sufficiently low energies it is proper to neglect cubic and higher terms in Eq. (2.9), and the system does indeed behave like a two-dimensional harmonic oscillator. It is somewhat surprising, however, that when $L^{\prime}=L_{T}^{\prime}$ (i.e., at exact 2:1 resonance), such cubic and higher terms cannot be neglected no matter how low the energy. In particular, the last term, $d z^{2}\left(\rho-\rho_{0}\right)$, produces an instability that is correlated with the bifurcation of the family of trajectories.

The regions of stability and instability of the periodic orbit can be calculated easily. Let us assume that the periodic orbit on the $\rho$ axis has a small amplitude about $\rho_{0}$. Then, at least for a while, the $\rho$ motion is to a good approximation harmonic, and

$$
\rho(t)=\rho_{0}(t)+A \cos \omega_{\rho}^{0} t .
$$

[Here $t$ has arbitrarily been set to zero at one of the maxima of $\rho(t)$.] To describe the $z$ motion, we must include the last term in (2.9),

$$
\frac{d^{2} z}{d t^{2}}=-\frac{\partial V}{\partial z}=-\left[k_{z}+d\left(\rho-\rho_{0}\right)\right] z
$$

With the approximation (6.1), this equation is

$$
\frac{d^{2} z}{d t^{2}}=-\left(k_{z}+A d \cos \omega_{\rho}^{0} t\right) z
$$

and with a change of variables,

$$
\tau=\frac{1}{2} \omega_{\rho}^{0} t
$$

it becomes

$$
\frac{d^{2} z}{d \tau^{2}}+(a-2 q \cos 2 \tau) z=0
$$

where

$$
\begin{aligned}
& a=4 k_{z} / \omega_{\rho}^{02}=4 k_{z} / k_{\rho}, \\
& -2 q=4 A d / \omega_{\rho}^{02} .
\end{aligned}
$$

Equation (6.5) is the standard form of Mathieu's equation. It describes a "parametrically driven" oscillator-a harmonic oscillator with a time-dependent and periodic force constant. The general theory of such equations is well-developed, ${ }^{12}$ and solutions to Mathieu's equation have been studied and tabulated. ${ }^{13}$ For almost all values of $q$ and $a$, the general solution to the equation can be written as a linear combination of two fundamental "Floquet solutions,"

$$
z(\tau)=C_{+} e^{\alpha \tau} P(\tau)+C_{-} e^{-\alpha \tau} P(-\tau) .
$$

The function $P(\tau)$ is periodic in $\tau$ with period $\pi$ (so it is periodic in $t$ with period $\left.2 \pi / \omega_{\rho}^{0}\right)$. Usually $P(-\tau)$ is independent of $P(\tau)$. The constant $\alpha$ is either real or purely imaginary, depending on the values of $q$ and $a$.
If $A$ is small, then $q$ is small, and for most values of $a$, $\alpha$ is purely imaginary. The $z$ motion is, therefore, multiply periodic, i.e., it can be described by a Fourier series with two fundamental frequencies, and harmonics of these frequencies.

$$
z(t)=\sum_{n_{1}, n_{2}} A_{n_{1} n_{2}} \exp \left[i\left(n_{1} \omega_{1}+n_{2} \omega_{2}\right) t\right] .
$$

One of the $\omega_{i}$ is exactly $\omega_{\rho}^{0}$, and the other is close to $\omega_{z}^{0}$. In this case, any initially small $z$ motion remains small, and therefore the periodic orbit on the $z$ axis is stable under small perturbations.

Completely different behavior is found for certain ranges of $a$, corresponding to near-resonances. If $q$ is small, and $a$ is close to $n^{2}$ where $n$ is an integer, it means that the natural frequency for motion in the $z$ direction is close to a multiple of half the natural frequency for motion in the $\rho$ direction

$$
\omega_{z}^{0} \simeq n \frac{1}{2} \omega_{\rho}^{0}
$$

(such a situation might be called a "subharmonic resonance"). In this situation $\alpha$ takes on real values. It follows that $z(t)$ has oscillations which grow exponentially with time. Near such resonances, therefore, the periodic orbit is unstable. In particular, the transition regime corresponds to $a \approx 1$, where the unstable $z$ oscillations are being driven by periodic motion in $\rho$, having about twice the frequency of the $z$ motion. (This type of "parametric resonance" is the process used by children to impart energy to a swing, by bending their knees in a rhythm corresponding to twice the natural frequency of the swing. ${ }^{14}$ )

At the boundary between stable and unstable motion in $z$, Mathieu's equation admits a periodic solution with period $\pi$ (and also a second solution that grows linearly with time). Given $q$, the value of $a$ corresponding to this boundary is regarded as an eigenvalue of the equation. These eigenvalues have been tabulated (they are related to eigenfunctions representing standing waves in an elliptical enclosure), and simple approximations for them are available. The two relevant eigenvalues are the ones closest to $a=1$; they are given by the formula

$$
a_{\mp}(q)-1=\mp q-\frac{q^{2}}{8} \pm \frac{q^{3}}{64}-\frac{q^{4}}{1526} \mp \frac{11 q^{5}}{36864} \ldots
$$

For $q$ small and $a_{-}(q)<a<a_{+}(q)$, the solutions to Mathieu's equation are unstable, while for $a<a_{-}(q)$ or $a>a_{+}(q)$, the solutions are stable. ${ }^{15}$

The phase of the fundamental Floquet solutions is also important. ${ }^{16}$ Considering the increasing Floquet solution $z_{+}(\tau)$ to Mathieu's equation having $C_{-}=0$ in Eq. (6.9). Consider those values of $q$ and $a$ such that $\alpha$ is real (that is, the unstable region). We want to know for what values of $\tau$ is $z_{+}(\tau)$ equal to zero? In this case $z(\tau)$ vanishes if and only if $P(\tau)$ vanishes. Now $P(\tau)$ is periodic in $\tau$ with period $\pi$ so we expect $P(\tau)$ to vanish twice in the interval $0 \leq \tau<\pi$. Also, from Eqs. (6.1) and (6.4),

$$
\rho(\tau)-\rho_{0}=A \cos 2 \tau
$$


is also periodic in $\tau$ with period $\pi$, and it vanishes when $\tau=\pi / 4$ or $3 \pi / 4$. Hence if we find the values of $\tau$ for which $P(\tau)$ vanishes ( $z$ vanishes), we are finding the phase of the growing Floquet solution $z_{+}(\tau)$ relative to the phase of $\rho(\tau)$.

A useful approximation to this phase has been given by Whittaker and Watson. ${ }^{13(a)}$ They give the formula

$$
z_{+}(\tau) \simeq e^{\alpha \tau} \sin (\tau-\sigma),
$$

where

$$
\sigma \simeq \frac{1}{2} \cos ^{-1}\left(\frac{1-a}{q}\right) .
$$

A more accurate version of this formula is given in Appendix B. In using Eqs. (6.11), we should also use the lowest-order version of (6.10),

$$
1-a_{\mp}= \pm q \text {. }
$$

It then follows that in the unstable region,

$$
|1-a| / q \leq 1
$$

and $\sigma$ calculated according to $(6.12)$ is real. (Whittaker and Watson did not specify which branch of $\cos ^{-1}$ is relevant; the appropriate value follows from the more complete analysis given in Appendix B.)

The meaning of Eq. (6.11) can be understood from the following remarks. Suppose we start with $a$ substantially greater than unity, in the stable region. We gradually decrease a until the periodic orbit first becomes unstable, at $a=a_{+}(q) \approx 1+q$, at which point $\sigma=\pi / 2$. Then the growing solution $z_{+}(\tau)$ oscillates like

$$
\sin (\tau-\pi / 2)=-\cos \tau \text {. }
$$

Hence $z_{+}(\tau)$ passes through zero at $\tau=\pi / 2+n \pi$, and at these values of $\tau, \rho-\rho_{0}=A \cos (2 \tau)=A \cos (\pi+2 n \pi)$ is a minimum. Similarly, if $a=1, z_{+}(\tau)$ passes through zero at the same time that $\rho-\rho_{0}$ passes through zero in the negative sense. Finally, if $a=a_{-}(q) \approx 1-q, z_{+}(\tau)$ passes through zero at the same time that $\rho-\rho_{0}$ is a maximum.

\section{B. Stability and bifurcations}

All of the above analysis is based upon the approximation (6.1) which leads to Mathieu's equation. This approximation can describe trajectories close to the periodic orbit for a finite length of time. (Since the amplitude of $\rho$ motion is fixed in this approximation but the amplitude of $z$ motion may grow exponentially, the approximation does not conserve energy, and after some finite time the exact trajectories must deviate from those computed by this approximation.) We now return to the exactly computed trajectories and ask, how do the properties of Mathieu's equation manifest themselves in the exact surfaces of section? We shall show that the bifurcation of the family of trajectories seen in the surface of section is correlated with the appearance of an unstable solution to Mathieu's equation.

To make the correlation, we need the interpretation of the Mathieu parameters $a$ and $q$ in terms of $L^{\prime}$ and $f$. From Eqs. (6.6) and (2.10), we find

$$
a=4 k_{z} / k_{\rho}=4\left(\frac{1}{\rho_{0}^{3}}\right) /\left(1+\frac{1}{\rho_{0}^{3}}\right) .
$$

This can be simplified to give

$$
a=4 /\left[1+\rho_{0}^{3}\left(L^{\prime}\right)\right] \text {. }
$$

Thus $a$ is directly related to $L^{\prime}$ [Eq. (2.7)]: in the transition regime it is a monotonically decreasing function of $L^{\prime}$. At $L^{\prime}=L_{T}^{\prime}, a=1$.

The parameter $q$ is related to the scaled energy $f$. In the harmonic-oscillator approximation (6.1), the energy of the periodic orbit is

$$
E=V\left(\rho_{0}\right)+\frac{1}{2} k_{\rho} A^{2},
$$

and from (6.7) we have

$$
A=-k_{\rho} q / 2 d
$$

so the relationship between the scaled energy $f$ of the exact trajectory and the parameter $q$ of Mathieu's equation is

$$
f\left(E_{s}-V_{0}\right)=E-V_{0}=\frac{1}{8} k_{\rho}^{3} q^{2} / d^{2}=A^{2} \frac{k_{\rho}}{2}
$$

(note that $k_{\rho}$ and $d$ depend upon $L^{\prime}$.) From Eqs. (6.13) and (6.16), given any values of $q$ and $a$ we can calculate $f$ and $L^{\prime}$, and vice versa.

Two observations constitute the fundamental conclusions of this section. Both apply if $f$ is not too large.

(1) The critical values $f$ and $L^{\prime}$ at which the exact trajectory field bifurcates into two families correspond closely to those values of $a$ and $q$ at which solutions of Mathieu's equation change from stable to unstable. Those values if $q$ and $a$ are the eigenvalues of Mathieu's equation, the relevant ones being given by Eq. (6.10). Combining that formula with Eqs. (6.13) and (6.16), we have calculated the values of $f$ and $L^{\prime}$ corresponding to the stability boundary for solutions to Mathieu's equation. The result is the solid curve shown in Fig. 7. Clearly this stability boundary for Mathieu's equation and the bifurcation boundary for the exact trajectories are very close together, especially for small $f$. At larger $f$, the harmonic-oscillator approximation (6.1) becomes less accurate, Mathieu's equation becomes less relevant as a comparison equation, and the bifurcation boundary begins to move away from the Mathieu stability boundary.

(2) The end points of the separatrix dividing the E family from the $\mathbf{H}$ family and correlated with the phases of the growing or decreasing Floquet solutions. According to Eq. (6.11), the growing Floquet solution passes through $z=0$ in the positive sense at times such that

$$
\tau=\tau^{*} \simeq \frac{1}{2} \cos ^{-1}\left(\frac{1-a}{q}\right) .
$$

At this value of $\tau$, according to Eq. (6.1), $\rho$ and $P_{\rho}$ are, respectively, 


$$
\begin{aligned}
& \rho=\rho_{0}+A \cos 2 \tau^{*}=\rho_{0}+A\left(\frac{1-a}{q}\right) \\
& =\rho_{0}\left(1+\rho_{0}^{3} / 3\right) / 2 \text {, } \\
& P_{\rho}=-\omega_{\rho}^{0} A \sin 2 \tau^{*} .
\end{aligned}
$$

The decreasing Floquet solution passes through zero in the positive sense at $\tau=-\tau^{*}$. The periodic orbit constitutes the outer boundary of the surface of section, and through Eqs. (6.18) the points $\pm \tau^{*}$ identify two specific points on it. Several composite surfaces of section were shown in Fig. 5, and the points corresponding to $\tau^{*}$ are marked; to within the resolution available in these pictures, $\tau^{*}$ corresponds to the position of the separatrix. In the figure the position is specified by the arrow, and the equations of Appendix B rather than Eqs. (6.18) are used. (Again as $f$ increases, some discrepancy between the observed and calculated position of the separatrix should appear.)

\section{CONCLUSIONS}

A one-electron atom in the presence of a magnetic field supports two families of trajectories in the transition regime near $L^{\prime}=1.6$. For a field of $1.88 \mathrm{~T}$ this corresponds to an $L_{z}$ value of about 80 , a value which appears to be attainable. Similar behavior can be expected for $\alpha \beta \approx 37 \frac{1}{2}$, which implies $L_{z} \approx 60$ and $B \approx 5 \mathrm{~T}$, which is more easily attained. If the hydrogenic atoms can be generated in the nearly circular excited states, then subjecting them to static magnetic fields of different intensity will reveal the avoided crossing. The ability to explore the resonant and near-resonant conditions by simply tuning the field is an important characteristic of this system.

We have shown that the bifurcation into two families is due to the existence of a 2:1 resonance in the frequencies of the $\rho$ and $z$ motions. A Floquet-Mathieu analysis in the resonance region demonstrates that at low energy the bifurcation boundary is accurately predicted by the instability boundary in the Mathieu analysis.

A semiclassical treatment accurately reproduces quantum variational eigenvalues through the resonance. However, due to the bifurcation, some semiclassical states may be missing, and others may be represented by trajectories from each family. The semiclassical eigentrajectories facilitate the interpretation of the transformation of the character of the wave functions through the transition zone. In particular, the semiclassical method reproduces the avoidance of crossing of eigenvalues. As we mentioned earlier, this result is different from that obtained by Noid and co-workers. We have no doubt about the correctness of their results, or of ours, so the difference must arise from the differences in the cases studied. The Hamiltonian studied in Ref. 3(a) was

$$
\begin{aligned}
H= & \frac{1}{2}\left(p_{x}^{2}+p_{y}^{2}\right)+\frac{1}{2}\left(\omega_{x}^{02} x^{2}+\omega_{y}^{02} y^{2}\right) \\
& -a\left(x^{3}+y^{3}\right)+\lambda x^{2} y^{2}-b x y^{3},
\end{aligned}
$$

with $\omega_{x}^{0}=3 \omega_{y}^{0}$ and the parameter varied was $\lambda$; the other parameters were held fixed. This system has an exact $3: 1$ resonance in the zeroth-order (quadratic) terms; a secular perturbation arises from the $x y^{3}$ term, and the other terms appear to produce small modifications to the trajectories. When the parameter $\lambda$ was varied, quantum eigenvalues showed avoid crossings, but primitive semiclassical eigenvalues crossed.

In our Hamiltonian, varying the parameter $\hat{L}$ or $L^{\prime}$ changes the coefficient of the term containing the secular perturbation, $\left(\rho-\rho_{0}\right) z^{2}$ in Eq. (2.9). More importantly, we think, variation of this parameter changes the zerothorder frequency ratio, so that we pass through a zone of zeroth-order resonance, from $\left(\omega_{x}^{0} / \omega_{y}^{0}\right)<2$ at small $L^{\prime}$ to $\left(\omega_{x}^{0} / \omega_{y}^{0}\right)>2$ at large $L^{\prime}$. This is quite different from the situation studied in Ref. 3(a), and it may explain the difference in our results.

\section{ACKNOWLEDGMENTS}

We acknowledge support of this research by a grant from the Jeffress Memorial Trust. Acknowledgment is made to the donors of the Petroleum Research Fund, administered by the American Chemical Society, for partial support of this research (S.K.K). J.B.D. acknowledges support from the National Science Foundation.

\section{APPENDIX A: CLASSICAL AND QUANTUM HAMILTONIANS, AND THE LANGER MODIFICATION}

The exact classical Hamiltonian for an electron interacting with a fixed nucleus in the presence of a magnetic field is

$$
H_{C}=\frac{1}{2 m_{e}}\left(P_{\rho}^{2}+P_{z}^{2}\right)+L_{z}^{2} / 2 m_{e} \rho^{2}+V(\rho, z) .
$$

The exact quantum Hamiltonian is

$H_{Q}=-\frac{\hbar^{2}}{2 m_{e}}\left[\frac{\partial^{2}}{\partial \rho^{2}}+\frac{1}{\rho} \frac{\partial}{\partial \rho}+\frac{\partial^{2}}{\partial z^{2}}\right]+\frac{m^{2} \hbar^{2}}{2 m_{e} \rho^{2}}+V(\rho, z)$

and the boundary conditions for bound states are

$$
\begin{aligned}
& \psi(\rho, z) \sim \rho^{m}, \quad \rho \rightarrow 0 \\
& \psi(\rho, z) \rightarrow 0, \quad \rho \rightarrow \infty \text { or }|z| \rightarrow \infty .
\end{aligned}
$$

If we define $\Phi(\rho, z)$ so that

$$
\psi(\rho, z)=\rho^{-1 / 2} \Phi(\rho, z)
$$

then the equation satisfied by $\Phi(\rho, z)$ is

$$
\left[-\frac{\hbar^{2}}{2 m_{e}}\left(\frac{\partial^{2}}{\partial \rho^{2}}+\frac{\partial^{2}}{\partial z^{2}}\right]+\frac{\left(m^{2}-\frac{1}{4}\right) \hbar^{2}}{2 m_{e} \rho^{2}}+V(\rho, z)\right] \Phi(\rho, z)=E \Phi(\rho, z)
$$


and the boundary condition (A3a) becomes

$$
\Phi(\rho, z) \sim \rho^{m+1 / 2}, \quad \rho \rightarrow 0 .
$$

Questions: If we use some sort of classical Hamiltonian in order to calculate a semiclassical approximation to eigenfunctions and eigenvalues, what is the best Hamiltonian to use? Does the exact classical Hamiltonian lead to the most accurate semiclassical results, or are there modifications of that classical Hamiltonian that can improve the results? Specifically, is it better to use $m^{2} \hbar^{2}$ or $\left(m^{2}-1 / 4\right) \hbar^{2}$ in the classical Hamiltonian? Should we also modify it by a term such as $(1 / \rho) P_{\rho}$ ?

For spherically symmetric systems, these questions were addressed by Langer in $1937 .{ }^{17} \mathrm{He}$ pointed out one source of inaccuracy in the semiclassical approximation for spherical systems. For $l \neq 0$, the actual boundary conditions are $\psi(r) \rightarrow 0$ as $r \rightarrow 0$. However, semiclassical connection formulas presume that the range of the variable (call it $x)$ is $(-\infty, \infty)$, and that the boundary condition on the wave function [call it $\bar{\psi}(x)$ ] is $\bar{\psi}(x) \rightarrow 0$ as $x \rightarrow-\infty$. He therefore proposed that the semiclassical approximation would be more accurate if we change the variable to $x=\ln r$. The effect of this change of variable is that the centrifugal term $l(l+1) \hbar^{2} / 2 m r^{2}$ is modified to $(l+1 / 2)^{2} \hbar^{2} / 2 m r^{2}$. He therefore concluded that improved accuracy would be obtained in the semiclassical approximation if this modification were made. Experimental evidence already had suggested this replacement, and since then his conclusion has been confirmed by careful comparisons of semiclassical approximations with exact quantum results.

The same question now arises with the Hamiltonian (A2), which is expressed in cylindrical coordinates. ${ }^{7}$ An especially difficult aspect of the problem is that the coordinates $(\rho, z)$ cannot be separated. To make progress, we ignore the $z$ motion, dropping $\partial^{2} / \partial z^{2}$ from the Hamiltonian, and suppressing the $z$ dependence of the potential energy.

Thus we consider the equation

$$
\left[-\frac{\hbar^{2}}{2 m_{e}}\left[\frac{\partial^{2}}{\partial \rho^{2}}+\frac{1}{\rho} \frac{\partial}{\partial \rho}\right]+\frac{m^{2} \hbar^{2}}{2 m_{e} \rho^{2}}+V(\rho)-E\right] \psi(\rho)=0 .
$$

If we make the substitution

$$
\psi(\rho)=\rho^{-1 / 2} \Phi(\rho),
$$

then $\Phi(\rho)$ obeys

$$
\left[-\frac{\hbar^{2}}{2 m_{e}} \frac{d^{2}}{d \rho^{2}}+V(\rho)+\frac{\left(m^{2}-\frac{1}{4}\right) \hbar^{2}}{2 m_{e} \rho^{2}}-E\right] \Phi(\rho)=0,
$$

subject to the boundary condition (A6). A WentzelKramers-Brillouin (WKB) approximation to $\Phi(\rho)$ leads to

$$
\psi(\rho) \simeq \rho^{-1 / 2} P^{*}(\rho)^{-1 / 2} \exp \left[i \int^{\rho} P^{*}\left(\rho^{\prime}\right) d \rho^{\prime} / \hbar\right),
$$

(A10a)

where
$P^{*}(\rho)=\left[2 m_{e}\left[E-V(\rho)-\frac{\left(m^{2}-\frac{1}{4}\right) \hbar^{2}}{2 m_{e} \rho^{2}}\right]\right]^{1 / 2}$.

This is one possible form of a WKB approximation.

An alternative WKB approximation is obtained by using the analogue of Langer's change of variables. It is most easily applied directly to (A7) rather than to (A9):

$$
\begin{aligned}
& x=\ln \rho, \\
& \rho=e^{x}, \\
& \psi(\rho)=\psi(\rho(x))=\bar{\psi}(x) .
\end{aligned}
$$

If $\psi(\rho)$ satisfies (A7), then $\bar{\psi}(x)$ satisfies

$$
\left[-\frac{\hbar^{2}}{2 m_{e}} \frac{d^{2}}{d x^{2}}+e^{2 x}\left[V(\rho(x))+\frac{m^{2} \hbar^{2}}{2 m_{e} \rho^{2}}-E\right] \bar{\psi}(x)=0\right.
$$

with the boundary condition $\bar{\psi}(x) \rightarrow 0$ as $x \rightarrow-\infty$ if $m \neq 0$. The WKB approximation for $\bar{\psi}(x)$ is

$$
\bar{\psi}(x) \simeq P_{1}(x)^{-1 / 2} \exp \left[i \int^{x} P_{1}\left(x^{\prime}\right) d x^{\prime} / \hbar\right),
$$

where

$$
P_{1}(x)=\left[2 m_{e} e^{2 x}\left[[E-V(\rho(x))]-\frac{m^{2} \hbar^{2}}{2 m_{e} \rho(x)^{2}}\right]\right]^{1 / 2}
$$

When this is reexpressed as a function of $\rho$ using (A8), we obtain

$\psi(\rho) \simeq \rho^{-1 / 2} P(\rho)^{-1 / 2} \exp \left[i \int^{\rho} P\left(\rho^{\prime}\right) d \rho^{\prime} / \hbar\right)$,

where

$P(\rho)=\left\{2 m_{e}\left[E-V(\rho)-\left(\frac{m^{2} \hbar^{2}}{2 m_{e} \rho^{2}}\right]\right)\right\}^{1 / 2}$.

The analogue of Langer's proposition is that (A14) is a better approximation to the exact quantum wave function than (A10). We believe this to be correct (though we note that for the cylindrical case this proposition has not yet been subjected to the careful scrutiny that it endured in the spherical case).

Returning to the full system (A2), we advance the hypothesis that the best semiclassical approximation to $\psi(\rho, z)$ is

$$
\psi(\rho, z) \simeq \rho^{-1 / 2} J^{-1 / 2} \exp (i S(\rho, z) / \hbar),
$$

where $S(\rho, z)$ and $J(\rho, z)$ are the classical action functions and the classical density Jacobians calculated according to the standard prescriptions, using an unmodified centrifugal barrier $m^{2} \hbar^{2} / 2 m_{e} \rho^{2}$.

The calculations performed in Sec. IV of this paper used similar concepts in a different way. We used the substitution (A4), arriving at the Eqs. (A5) and (A6). However, in our quantum calculations, we allowed a little inaccuracy, by ignoring the boundary condition (A6) and 
using instead the condition $\Phi(\rho, z) \rightarrow 0$ as $\rho \rightarrow-\infty$. Then in our semiclassical calculations, we want to use that classical Hamiltonian which will give the closest possible correspondence with our (slightly inaccurate) quantum results. Therefore in our classical Hamiltonian we should take the centrifugal barrier to be $\left(m^{2}-\frac{1}{4}\right) \hbar^{2} / 2 m_{e} \rho^{2}$. This is how the calculations were performed. We should repeat that while the formal comparison between classical and quantum Hamiltonians forces us to distinguish between $m^{2}$ and $m^{2}-\frac{1}{4}$, and also forces us to examine boundary conditions carefully; in the present case these distinctions are negligible. For example, if $\hat{L}=1.6$, then $L^{\prime} \equiv\left(\hat{L}^{2}-\hat{\hbar}^{2} / 4\right)^{1 / 2}=1.5997$, so the difference is in the fifth significant digit.

\section{APPENDIX B: AN IMPROVED APPROXIMATION FOR THE END POINTS OF THE SEPARATRIX}

In Sec. IV, we suggested that there is a close relationship between the separatrix associated with the exact equations of motion and the phase of the exponentially growing (or decreasing) solution of Mathieu's equation. Equations (6.17) and (6.18) give an approximate evaluation of that phase. Here we present formulas that give a more precise evaluation of the phase. All the required formulas are derived by Whittaker and Watson, and we only have to adapt their results to our notation.

Mathieu's equation is written in the form (6.5), and we take $a$ and $q$ to be given. For comparison with solutions to the exact equations of motion, $a$ and $q$ are calculated from $L^{\prime}$ and $f$ using Eqs. (6.13) and (6.16). From $a$ and $q$, an auxiliary parameter $\sigma$ is defined as the solution to the equation

$$
\begin{aligned}
a= & 1-q \cos 2 \sigma+\left(-\frac{1}{4}+\frac{1}{8} \cos 4 \sigma\right) q^{2}+\frac{1}{64} q^{3} \cos 2 \sigma \\
& +\left(\frac{1}{48}-\frac{11}{512} \cos 4 \sigma\right) q^{4}+\cdots
\end{aligned}
$$

(higher-order terms are neglected). After $\sigma$ is evaluated, then the following coefficients must be computed:

$$
\begin{aligned}
a_{3}= & 3(q / 8)^{2} \sin 2 \sigma-3(q / 8)^{3} \sin 4 \sigma \\
& +(q / 8)^{4}\left(-\frac{274}{9} \sin 2 \sigma+9 \sin 6 \sigma\right)+\cdots \\
b_{3}= & -(q / 8)+(q / 8)^{2} \cos 2 \sigma \\
& -(q / 8)^{3}\left(-\frac{14}{3}+5 \cos 4 \sigma\right) \\
& +(q / 8)^{4}\left(-\frac{74}{9} \cos 2 \sigma+7 \cos 6 \sigma\right)+\cdots, \\
a_{5}= & -\left(\frac{14}{9}\right)\left(\frac{q}{8}\right)^{3} \sin 2 \sigma+\frac{44}{27}\left(\frac{q}{8}\right)^{4} \sin 4 \sigma, \\
b_{5}= & \frac{1}{3}(q / 8)^{2}-\frac{4}{9}(q / 8)^{3} \cos 2 \sigma \\
& +(q / 8)^{4}\left(-\frac{155}{54}+\frac{82}{27} \cos 4 \sigma\right) \\
a_{7}= & \frac{35}{108}(q / 8)^{4} \sin 2 \sigma, \\
b_{7}= & -\frac{1}{18}(q / 8)^{3}+\frac{1}{12}(q / 8)^{4} \cos 2 \sigma \\
b_{9}= & \frac{1}{180}(q / 8)^{4} .
\end{aligned}
$$

The function $P(\tau)$ is given by a series,

$$
\begin{aligned}
P(\tau)=\sin (\tau-\sigma) & +a_{3} \cos (3 \tau-\sigma)+b_{3} \sin (3 \tau-\sigma) \\
& +a_{5} \cos (5 \tau-\sigma)+b_{5} \sin (5 \tau-\sigma) \\
& +a_{7} \cos (7 \tau-\sigma)+b_{7} \sin (7 \tau-\sigma) \\
& +b_{9} \sin (9 \tau-\sigma)+\ldots .
\end{aligned}
$$

The relevant phase is the value of $\tau^{*}$ such that $P\left(\tau^{*}\right)=0$.

After $\tau^{*}$ has been calculated, a point on the surface of section is identified using Eqs. (6.18). We observe that this point is very close to the endpoint of the separatrix arising from the exact trajectory equations.
${ }^{1}$ (a) J. B. Delos, S. K. Knudson, and D. W. Noid, Phys. Rev. Lett. 50, 579 (1983); (b) Phys. Rev. A 28, 7 (1983); (c) 30, 1208 (1984); (d) D. W. Noid, S. K. Knudson, and J. B. Delos, Chem. Phys. Lett. 100, 367 (1983); (e) R. L. Waterland, J. B. Delos, and M. L. Du, Phys. Rev. A 35, 5064 (1987); (f) J. B. Delos, R. L. Waterland, and M. L. Du, Phys. Rev. A 37, 1185 (1988); (g) M. L. Du and J. B. Delos (unpublished).

${ }^{2}$ G. Herzberg, Molecular Spectra and Structure II (Van Nostrand, Princeton, NJ, 1945), p. 218.

${ }^{3}$ (a) D. W. Noid, M. L. Koszykowski, and R. A. Marcus, J. Chem. Phys. 78, 4018 (1983); (b) 71, 2864 (1979); (c) D. W. Noid and R. A. Marcus, ibid. 85, 3305 (1986); (d) T. Uzer, D. W. Noid and R. A. Marcus, ibid. 79, 4412 (1983); (e) D. Farrelly and T. Uzer, ibid. 85, 308 (1986).

${ }^{4}$ M. E. Kellman and Eric D. Lynch, J. Chem. Phys. 85, 7216 (1986); G. A. Voth and R. A. Marcus, ibid. 82, 4064 (1985); E. J. Heller, E. B. Stechel, and M. J. Davis, ibid. 73, 4720 (1980); M. J. Davis and E. J. Heller, ibid. 80, 5036 (1984); N. DeLeon, M. J. Davis, and E. J. Heller, ibid. 80, 794 (1984); E. J. Heller and W. M. Gelbart, ibid. 73, 626 (1980); R. B. Shirts, Chem. Phys. 114, 187 (1987). 5(a) D. W. Noid and R. A. Marcus, J. Chem. Phys. 62, 2119 (1975); (b) D. W. Noid, M. L. Koszykowski, and R. A. Marcus, Annu. Rev. Phys. Chem. 32, 267 (1981).

6(a) E. Thiele and J. Stone, J. Chem. Phys. 83, 312 (1985); (b) E. Thiele, M. F. Goodman, and J. Stone, ibid. 82, 2598 (1985).

${ }^{7}$ Langer-type corrections have been discussed in several other papers. J. A. C. Gallas and R. F. O'Connell, J. Phys. B 15, L593 (1982) appear to use the same method and arrive at the same conclusions as obtained here. D. Farrelly and W. P. Reinhardt, J. Phys. B 16, 2103 (1983) claim that the Langer correction appears "automatically" by looking at the Hamilton-Jacobi equation instead of the Schrödinger equation; however, the essential step in their derivation is the statement "Clearly, $\ldots P_{\phi}=m \ldots$. . R. J. Fonck, F. L. Roesler, D. H. Tracy, and F. S. Tomkins, Phys. Rev. A 21, 861 (1980) replace $m^{2}-\frac{1}{4}$ by $m^{2}$ “. . . in order that the WBK approximation be valid for all $\rho>0$." J. E. Adams and W. H. Miller, J. Chem. Phys. 67, 5775 (1977) give a general discussion of Langer-type corrections, but they do not specifically consider the cylindrical case. Finally, M. A. Collins, J. Chem. Phys. 85, 3902 (1986), has presented an analysis which 
leads to the surprising conclusion that in semiclassical mechanics $p_{\phi} \equiv L_{z}$ should be quantized as $\left(m+\frac{1}{2}\right) \hbar$. Our analysis does not agree with this.

${ }^{8}$ L. F. Shampine and M. K. Gordon, Computer Solutions of Ordinary Differential Equations (Freeman, San Franscisco, 1975).

${ }^{9}$ M. Born, Mechanics of the Atom (Ungar, New York, 1960).

${ }^{10}$ S. K. Knudson, D. W. Noid, and M. L. Koszykowski, J. Phys. Chem. 91, 2763 (1987).

${ }^{11}$ (a) J. B. Delos, Adv. Chem. Phys. 65, 161 (1986); (b) S. K. Knudson, J. B. Delos, and B. Bloom, J. Chem. Phys. 83, 5703 (1985); (c) S. K. Knudson, J. B. Delos, and D. W. Noid, ibid. 84, 6886 (1986).

${ }^{12}$ E. L. Ince, Ordinary Differential Equations (Dover, New York, 1944).
${ }^{13}$ (a) E. T. Whittaker and G. N. Watson, A Course of Modern Analysis (Cambridge University Press, Cambridge, England, 1963); (b) Handbook of Mathematical Functions, edited by M. Abramowitz and E. Stegun (U.S. GPO, Washington, D.C., 1972).

${ }^{14}$ P. L. Tea, Jr. and H. Falk, Am. J. Phys. 36, 1165 (1968); see also B. F. Gore, ibid. 38, 378 (1970); J. A. Burns, ibid. 38, 920 (1970); J. T. McMullan, ibid. 40, 764 (1972); B. F. Gore, ibid. 39, 347 (1971); A. E. Siegman, ibid. 37, 843 (1969).

${ }^{15}$ This statement describes the first unstable zone. Other unstable zones in the Mathieu equation occur at $a \approx 4, a \approx 9$, $a \approx 16$, etc.

${ }^{16}$ Here we go beyond the analysis given in Ref. 6 .

${ }^{17}$ R. E. Langer, Phys. Rev. 51, 669 (1937). 

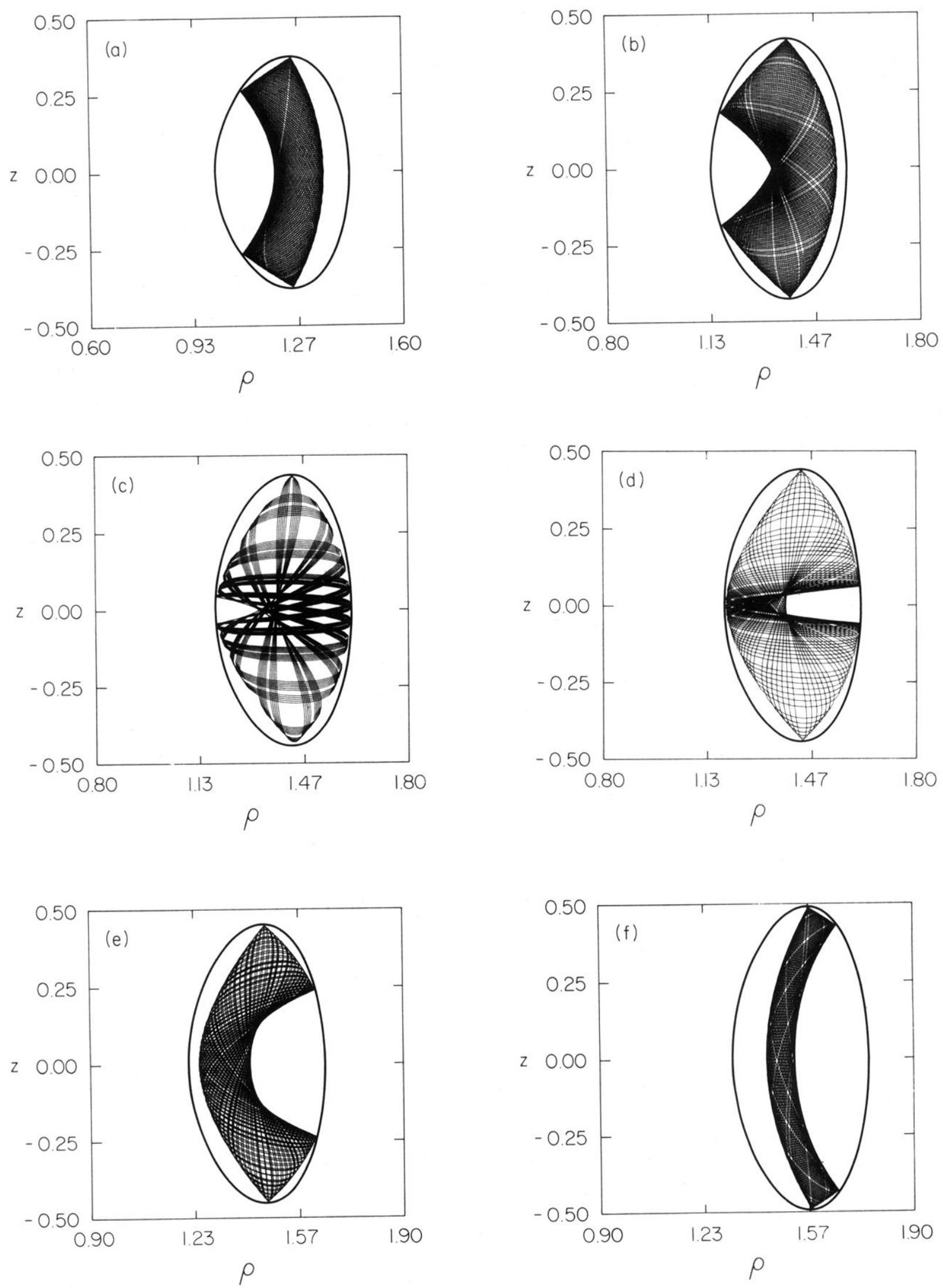

FIG. 8. A sequence of trajectories with initial conditions $f=0.05, z^{I}=0, P_{\rho}^{I}=0, \rho^{I}=\rho_{0}$, for increasing $\rho_{0}$, corresponding to increasing $L$. The sequence shows the correlation between trajectories belonging to different families. (a) $\hat{L}=1.3$; (b) $\hat{L}=1.45$; (c) $\hat{L}=1.510$; (d) $\hat{L}=1.515$; (e) $\hat{L}=1.55$; and (f) $\hat{L}=1.70$. 

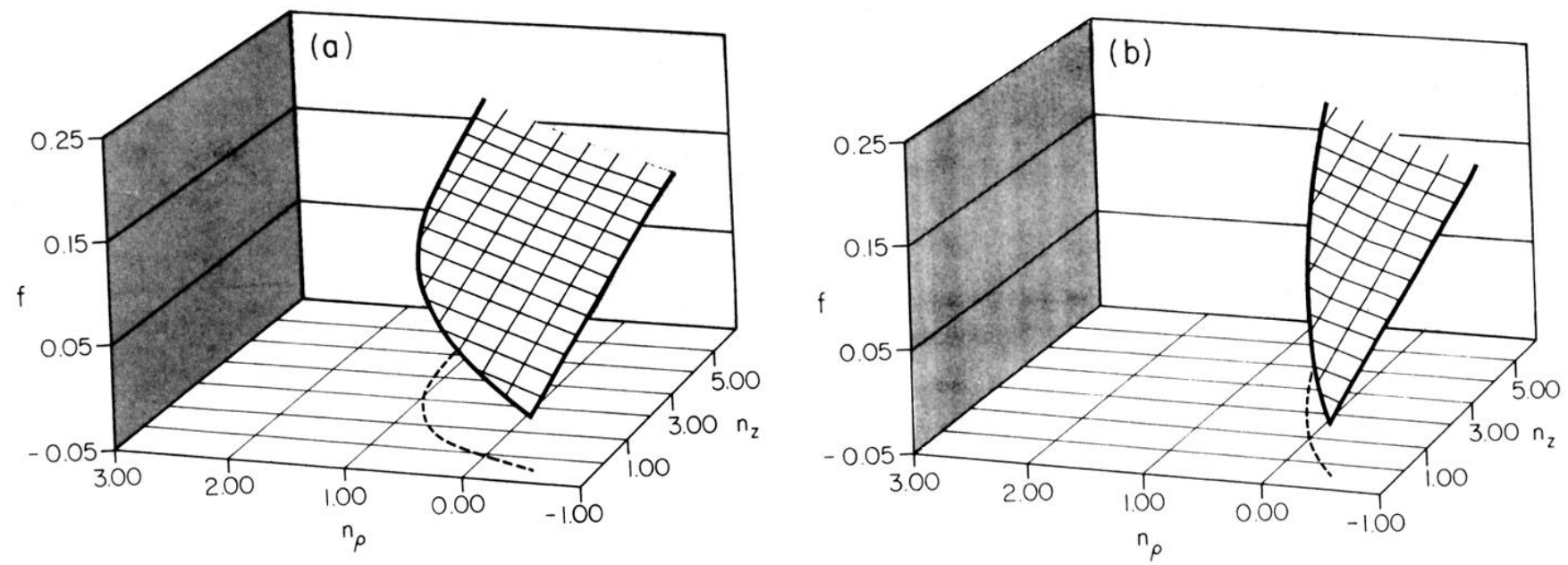

FIG. 9. (a) The surface $f_{\mathrm{H}}\left(n_{\rho}, n_{z}\right)$ in which the "quantum numbers" are regarded as continuous variables. The values of the surface at integer grid intersections are the semiclassical eigenvalues. The dotted line in the $\left(n_{\rho}, n_{z}\right)$ plane locates the minimum value of $n_{z}$ at each given value of $n_{\rho}$. (b) as in (a), but for $f_{\mathrm{E}}\left(n_{\rho}, n_{z}\right)$. 